\title{
The truncated Euler-Maruyama method for stochastic differential delay equations
}

\author{
Qian Guo $^{1} \cdot$ Xuerong Mao' ${ }^{1,2}$ (D) Rongxian Yue $^{1}$
}

Received: 8 June 2016 / Accepted: 31 July 2017 / Published online: 11 August 2017

(C) The Author(s) 2017. This article is an open access publication

\begin{abstract}
The numerical solutions of stochastic differential delay equations (SDDEs) under the generalized Khasminskii-type condition were discussed by Mao (Appl. Math. Comput. 217, 5512-5524 2011), and the theory there showed that the Euler-Maruyama (EM) numerical solutions converge to the true solutions in probability. However, there is so far no result on the strong convergence (namely in $L^{p}$ ) of the numerical solutions for the SDDEs under this generalized condition. In this paper, we will use the truncated EM method developed by Mao (J. Comput. Appl. Math. 290, 370-384 2015) to study the strong convergence of the numerical solutions for the SDDEs under the generalized Khasminskii-type condition.
\end{abstract}

Keywords Brownian motion · Stochastic differential delay equation · Itô's formula $\cdot$ Truncated Euler-Maruyama $\cdot$ Khasminskii-type condition

Mathematical subject classifications (2000) $60 \mathrm{H} 10 \cdot 60 \mathrm{~J} 65$

\section{Introduction}

In the study of stochastic differential delay equations (SDDEs), the classical existence-and-uniqueness theorem requires that the coefficients of the SDDEs satisfy the local Lipschitz condition and the linear growth condition (see, e.g., [1, 6, $9,12,20])$. However, there are many SDDEs which do not satisfy the linear growth

Xuerong Mao

x.mao@strath.ac.uk

1 Department of Mathematics, Shanghai Normal University, Shanghai, China

2 Department of Mathematics and Statistics, University of Strathclyde, Glasgow G1 1XH, UK 
condition. In 2002, Mao [14] generalized the the well-known Khasminskii test [7] from stochastic differential equations (SDEs) to SDDEs. The Khasminskii-type theorem established in [14] for SDDEs gives the conditions, in terms of Lyapunov functions, under which the solutions to SDDEs will not explode to infinity at a finite time. The Khasminskii-type theorem enables us to verify if a given nonlinear SDDE has a unique global solution under the local Lipschitz condition but without the linear growth condition. In 2005, Mao and Rassias [17] demonstrated that there are many important SDDEs which are not covered by the Khasminskii-type theorem given in [14] and established a generalized Khasminskii-type theorem which covers a very wide class of nonlinear SDDEs.

On the other hand, there are in general no explicit solutions to nonlinear SDDEs, whence numerical solutions are required in practice. The numerical solutions under the linear growth condition plus the local Lipschitz condition have been discussed intensively by many authors (see, e.g., [3-5, 8, 11, 19, 21]). The numerical solutions of SDDEs under the generalized Khasminskii-type condition were discussed by Mao [15], and the theory there showed that the Euler-Maruyama (EM) numerical solutions converge to the true solutions in probability. However, there is so far no result on the strong convergence (namely in $L^{p}$ ) of the numerical solutions for the SDDEs under the generalized Khasminskii-type condition.

Recently, Mao [16] develops a new explicit numerical method, called the truncated EM method, for SDEs under the Khasminskii-type condition plus the local Lipschitz condition and establishes the strong convergence theory. In this paper, we will use this new truncated EM method to study the strong convergence of the numerical solutions for the SDDEs under the generalized Khasminskii-type condition.

This paper is organized as follows: We will introduce necessary notion, state the generalized Khasminskii-type condition, and define the truncated EM numerical solutions for SDDEs in Section 2. We will establish the strong convergence theory for the truncated EM numerical solutions in Sections 3 and 4 and discuss the convergence rates in Section 5. In each of these three sections, we will illustrate our theory by examples. We will see from these examples that the truncated EM numerical method can be applied to approximate the solutions of many highly nonlinear SDDEs. We will finally conclude our paper in Section 6.

\section{The truncated Euler-Maruyama method}

Throughout this paper, unless otherwise specified, we use the following notation. Let $|\cdot|$ be the Euclidean norm in $\mathbb{R}^{n}$. If $A$ is a vector or matrix, its transpose is denoted by $A^{T}$. If $A$ is a matrix, its trace norm is denoted by $|A|=\sqrt{\operatorname{trace}\left(A^{T} A\right)}$. Let $\mathbb{R}_{+}=[0, \infty)$ and $\tau>0$. Denote by $C\left([-\tau, 0] ; \mathbb{R}^{n}\right)$ the family of continuous functions from $[-\tau, 0]$ to $\mathbb{R}^{n}$ with the norm $\|\varphi\|=\sup _{-\tau \leq \theta \leq 0}|\varphi(\theta)|$. Let $\left(\Omega, \mathcal{F},\left\{\mathcal{F}_{t}\right\}_{t \geq 0}, \mathbb{P}\right)$ be a complete probability space with a filtration $\left\{\mathcal{F}_{t}\right\}_{t \geq 0}$ satisfying the usual conditions (i.e., it is increasing and right continuous while $\mathcal{F}_{0}$ contains all $\mathbb{P}$-null sets). Let $B(t)=\left(B_{1}(t), \cdots, B_{m}(t)\right)^{T}$ be an $m$-dimensional Brownian motion defined on the probability space. Moreover, for two real numbers $a$ and $b$, we use $a \vee b=\max (a, b)$ and $a \wedge b=\min (a, b)$. If $G$ is a set, its indicator function is 
denoted by $I_{G}$, namely $I_{G}(x)=1$ if $x \in G$ and 0 otherwise. If $a$ is a real number, we denote by $\lfloor a\rfloor$ the largest integer which is less or equal to $a$, e.g., $\lfloor-1.2\rfloor=-2$ and $\lfloor 2.3\rfloor=2$.

Consider a nonlinear SDDE

$$
d x(t)=f(x(t), x(t-\tau)) d t+g(x(t), x(t-\tau)) d B(t), \quad t \geq 0,
$$

with the initial data given by

$$
\{x(\theta):-\tau \leq \theta \leq 0\}=\xi \in C\left([-\tau, 0] ; \mathbb{R}^{n}\right) .
$$

Here

$$
f: \mathbb{R}^{n} \times \mathbb{R}^{n} \rightarrow \mathbb{R}^{n} \quad \text { and } \quad g: \mathbb{R}^{n} \times \mathbb{R}^{n} \rightarrow \mathbb{R}^{n \times m} .
$$

We assume that the coefficients $f$ and $g$ obey the Local Lipschitz condition:

Assumption 2.1 For every positive number $R$, there is a positive constant $K_{R}$ such that

$$
|f(x, y)-f(\bar{x}, \bar{y})|^{2} \vee|g(x, y)-g(\bar{x}, \bar{y})|^{2} \leq K_{R}\left(|x-\bar{x}|^{2}+|y-\bar{y}|^{2}\right)
$$

for those $x, y, \bar{x}, \bar{y} \in \mathbb{R}^{n}$ with $|x| \vee|y| \vee|\bar{x}| \vee|\bar{y}| \leq R$.

The classical existence-and-uniqueness theorem does not only require this local Lipschitz condition but also the linear growth condition (see, e.g., [10, 12, 13, 20]). In this paper, we shall retain the local Lipschitz condition but replace the linear growth condition by a generalized Khasminskii-type condition.

Assumption 2.2 There are constants $K_{1}>0, K_{2} \geq 0$ and $\beta>2$ such that

$$
x^{T} f(x, y)+\frac{1}{2}|g(x, y)|^{2} \leq K_{1}\left(1+|x|^{2}+|y|^{2}\right)-K_{2}|x|^{\beta}+K_{2}|y|^{\beta}
$$

for all $(x, y) \in \mathbb{R}^{n} \times \mathbb{R}^{n}$.

To have a feeling about what type of nonlinear SDDEs to which our theory may apply, please consider, for example, the scalar SDDE

$d x(t)=\left[a_{1}+a_{2}|x(t-\tau)|^{4 / 3}-a_{3} x^{3}(t)\right] d t+\left[a_{4}|x(t)|^{3 / 2}+a_{5} x(t-\tau)\right] d B(t), \quad t \geq 0$, where $a_{3}>0$ and $a_{1}, a_{2}, a_{4}, a_{5} \in \mathbb{R}$ (see Example 4.8 for the details) or the 2dimensional SDDE in Example 3.7. The following result, established in [17], is a generalized Khasminskii-type theorem on the existence and uniqueness of the solution to the SDDE.

Lemma 2.3 Let Assumptions 2.1 and 2.2 hold. Then, for any given initial data (2.2), there is a unique global solution $x(t)$ to (2.1) on $t \in[-\tau, \infty)$. Moreover, the solution has the property that

$$
\sup _{-\tau \leq t \leq T} \mathbb{E}|x(t)|^{2}<\infty, \quad \forall T>0
$$

It has been shown (see, e.g., [15]) that under Assumptions 2.1 and 2.2, the EM numerical solutions converge to the true solution in probability. But, to our best 
knowledge, there is so far no result on the strong convergence under these assumptions. In this paper, we will use the truncated EM method developed in [16] and show that the truncated EM solutions will converge to the true solution in $L^{q}$ for some $q \geq 1$.

To define the truncated EM numerical solutions, we first choose a strictly increasing continuous function $\mu: \mathbb{R}_{+} \rightarrow \mathbb{R}_{+}$such that $\mu(r) \rightarrow \infty$ as $r \rightarrow \infty$ and

$$
\sup _{|x| \vee|y| \leq r}(|f(x, y)| \vee|g(x, y)|) \leq \mu(r), \quad \forall r \geq 1 .
$$

Denoted by $\mu^{-1}$ is the inverse function of $\mu$ and we see that $\mu^{-1}$ is a strictly increasing continuous function from $[\mu(0), \infty)$ to $\mathbb{R}_{+}$. We also choose a constant $\Delta^{*} \in(0,1]$ and a strictly decreasing function $h:\left(0, \Delta^{*}\right] \rightarrow(0, \infty)$ such that

$$
h\left(\Delta^{*}\right) \geq \mu(1), \quad \lim _{\Delta \rightarrow 0} h(\Delta)=\infty \quad \text { and } \quad \Delta^{1 / 4} h(\Delta) \leq 1, \quad \forall \Delta \in\left(0, \Delta^{*}\right] .
$$

For example, we may choose $\Delta^{*} \in(0,1)$ sufficiently small such that $1 / \Delta^{*} \geq$ $(\mu(1))^{4}$ and define $h(\Delta)=\Delta^{-1 / 4}$ for $\Delta \in\left(0, \Delta^{*}\right]$. For a given step size $\Delta \in$ $\left(0, \Delta^{*}\right]$, let us define a mapping $\pi_{\Delta}$ from $\mathbb{R}^{n}$ to the closed ball $\left\{x \in \mathbb{R}^{n}:|x| \leq\right.$ $\left.\mu^{-1}(h(\Delta))\right\}$ by

$$
\pi_{\Delta}(x)=\left(|x| \wedge \mu^{-1}(h(\Delta))\right) \frac{x}{|x|},
$$

where we set $x /|x|=0$ when $x=0$. That is, $\pi_{\Delta}$ will map $x$ to itself when $|x| \leq$ $\mu^{-1}(h(\Delta))$ and to $\mu^{-1}(h(\Delta)) x /|x|$ when $|x|>\mu^{-1}(h(\Delta))$. We then define the truncated functions

$$
f_{\Delta}(x, y)=f\left(\pi_{\Delta}(x), \pi_{\Delta}(y)\right) \quad \text { and } \quad g_{\Delta}(x, y)=g\left(\pi_{\Delta}(x), \pi_{\Delta}(y)\right)
$$

for $x, y \in \mathbb{R}^{n}$. It is easy to see that

$$
\left|f_{\Delta}(x, y)\right| \vee\left|g_{\Delta}(x, y)\right| \leq \mu\left(\mu^{-1}(h(\Delta))\right)=h(\Delta), \quad \forall x, y \in \mathbb{R}^{n} .
$$

That is, both truncated functions $f_{\Delta}$ and $g_{\Delta}$ are bounded although $f$ and $g$ may not. More usefully, these truncated functions preserve the generalized Khasminskii-type condition to a very nice degree as described in the following lemma.

Lemma 2.4 Let Assumption 2.2 hold. Then, for every $\Delta \in\left(0, \Delta^{*}\right]$, we have

$$
x^{T} f_{\Delta}(x, y)+\frac{1}{2}\left|g_{\Delta}(x, y)\right|^{2} \leq 2 K_{1}\left(1+|x|^{2}+|y|^{2}\right)-K_{2}\left|\pi_{\Delta}(x)\right|^{\beta}+K_{2}\left|\pi_{\Delta}(y)\right|^{\beta}
$$

for all $x, y \in \mathbb{R}^{n}$.

Proof Fix any $\Delta \in\left(0, \Delta^{*}\right]$. Recalling that $h\left(\Delta^{*}\right) \geq \mu(1)$, we see that $\mu^{-1}\left(h\left(\Delta^{*}\right)\right) \geq 1$. But $h$ is decreasing while $\mu^{-1}$ is increasing, so $\mu^{-1}(h(\Delta)) 4$ $\geq 1$. 
For $x \in \mathbb{R}^{n}$ with $|x| \leq \mu^{-1}(h(\Delta))$ and any $y \in \mathbb{R}^{n}$, we have, by (2.3),

$$
\begin{aligned}
& x^{T} f_{\Delta}(x, y)+\frac{1}{2}\left|g_{\Delta}(x, y)\right|^{2} \\
= & \pi_{\Delta}(x)^{T} f\left(\pi_{\Delta}(x), \pi_{\Delta}(y)\right)+\frac{1}{2}\left|g\left(\pi_{\Delta}(x), \pi_{\Delta}(y)\right)\right|^{2} \\
\leq & K_{1}\left(1+\left|\pi_{\Delta}(x)\right|^{2}+\left|\pi_{\Delta}(y)\right|^{2}\right)-K_{2}\left|\pi_{\Delta}(x)\right|^{\beta}+K_{2}\left|\pi_{\Delta}(y)\right|^{\beta} \\
\leq & K_{1}\left(1+|x|^{2}+|y|^{2}\right)-K_{2}\left|\pi_{\Delta}(x)\right|^{\beta}+K_{2}\left|\pi_{\Delta}(y)\right|^{\beta},
\end{aligned}
$$

which implies the desired assertion (2.9). On the other hand, for $x \in \mathbb{R}^{n}$ with $|x|>$ $\mu^{-1}(h(\Delta))$ and any $y \in \mathbb{R}^{n}$, we have

$$
\begin{aligned}
& x^{T} f_{\Delta}(x, y)+\frac{1}{2}\left|g_{\Delta}(x, y)\right|^{2} \\
= & \pi_{\Delta}(x)^{T} f\left(\pi_{\Delta}(x), \pi_{\Delta}(y)\right)+\frac{1}{2}\left|g\left(\pi_{\Delta}(x), \pi_{\Delta}(y)\right)\right|^{2} \\
& +\left(x-\pi_{\Delta}(x)\right)^{T} f\left(\pi_{\Delta}(x), \pi_{\Delta}(y)\right) \\
\leq & K_{1}\left(1+\left|\pi_{\Delta}(x)\right|^{2}+\left|\pi_{\Delta}(y)\right|^{2}\right)-K_{2}\left|\pi_{\Delta}(x)\right|^{\beta}+K_{2}\left|\pi_{\Delta}(y)\right|^{\beta} \\
& +\left(\frac{|x|}{\mu^{-1}(h(\Delta))}-1\right) \pi_{\Delta}(x)^{T} f\left(\pi_{\Delta}(x), \pi_{\Delta}(y)\right),
\end{aligned}
$$

where (2.3) has been used. But once again we see from (2.3) that

$$
\begin{aligned}
& \pi_{\Delta}(x)^{T} f\left(\pi_{\Delta}(x), \pi_{\Delta}(y)\right) \\
\leq & K_{1}\left(1+\left|\pi_{\Delta}(x)\right|^{2}+\left|\pi_{\Delta}(y)\right|^{2}\right)-K_{2}\left[\mu^{-1}(h(\Delta))\right]^{\beta}+K_{2}\left|\pi_{\Delta}(y)\right|^{\beta} \\
\leq & K_{1}\left(1+\left|\pi_{\Delta}(x)\right|^{2}+\left|\pi_{\Delta}(y)\right|^{2}\right),
\end{aligned}
$$

where we have used the property $\left|\pi_{\Delta}(y)\right| \leq \mu^{-1}(h(\Delta))$ by the definition of $\pi(\cdot)$. Substituting this into (2.11) yields

$$
\begin{aligned}
& x^{T} f_{\Delta}(x, y)+\frac{1}{2}\left|g_{\Delta}(x, y)\right|^{2} \\
\leq & \frac{K_{1}|x|}{\mu^{-1}(h(\Delta))}\left(1+\left|\pi_{\Delta}(x)\right|^{2}+\left|\pi_{\Delta}(y)\right|^{2}\right)-K_{2}\left|\pi_{\Delta}(x)\right|^{\beta}+K_{2}\left|\pi_{\Delta}(y)\right|^{\beta} \\
\leq & K_{1}|x|(1+|x|+|y|)-K_{2}\left|\pi_{\Delta}(x)\right|^{\beta}+K_{2}\left|\pi_{\Delta}(y)\right|^{\beta} \\
\leq & 2 K_{1}\left(1+|x|^{2}+|y|^{2}\right)-K_{2}\left|\pi_{\Delta}(x)\right|^{\beta}+K_{2}\left|\pi_{\Delta}(y)\right|^{\beta} .
\end{aligned}
$$

Namely, we have showed that the required assertion (2.9) also holds for $x \in \mathbb{R}^{n}$ with $|x|>\mu^{-1}(h(\Delta))$ and any $y \in \mathbb{R}^{n}$. The proof is hence complete.

From now on, we will let the step size $\Delta$ be a fraction of $\tau$. That is, we will use $\Delta=\tau / M$ for some positive integer $M$. When we use the terms of a sufficiently small $\Delta$, we mean that we choose $M$ sufficiently large.

Let us now form the discrete-time truncated EM solutions. Define $t_{k}=k \Delta$ for $k=$ $-M,-(M-1), \cdots, 0,1,2, \cdots$. Set $X_{\Delta}\left(t_{k}\right)=\xi\left(t_{k}\right)$ for $k=-M,-(M-1), \cdots, 0$ and then form

$$
X_{\Delta}\left(t_{k+1}\right)=X_{\Delta}\left(t_{k}\right)+f_{\Delta}\left(X_{\Delta}\left(t_{k}\right), X_{\Delta}\left(t_{k-M}\right)\right) \Delta+g_{\Delta}\left(X_{\Delta}\left(t_{k}\right), X_{\Delta}\left(t_{k-M}\right)\right) \Delta B_{k}
$$


for $k=0,1,2, \cdots$, where $\Delta B_{k}=B\left(t_{k+1}\right)-B\left(t_{k}\right)$. In our analysis, it is more convenient to work on the continuous-time approximations. There are two continuous-time versions. One is the continuous-time step process $\bar{x}_{\Delta}(t)$ on $t \in[-\tau, \infty)$ defined by

$$
\bar{x}_{\Delta}(t)=\sum_{k=-M}^{\infty} X_{\Delta}\left(t_{k}\right) I_{[k \Delta,(k+1) \Delta)}(t),
$$

where $I_{[k \Delta,(k+1) \Delta)}(t)$ is the indicator function of $[k \Delta,(k+1) \Delta)$ (please recall the notation defined in the beginning of this section). The other one is the continuoustime continuous process $x_{\Delta}(t)$ on $t \in[-\tau, \infty)$ defined by $x_{\Delta}(t)=\xi(t)$ for $t \in$ $[-\tau, 0]$ while for $t \geq 0$

$$
x_{\Delta}(t)=\xi(0)+\int_{0}^{t} f_{\Delta}\left(\bar{x}_{\Delta}(s), \bar{x}_{\Delta}(s-\tau)\right) d s+\int_{0}^{t} g_{\Delta}\left(\bar{x}_{\Delta}(s), \bar{x}_{\Delta}(s-\tau)\right) d B(s) .
$$

We see that $x_{\Delta}(t)$ is an Itô process on $t \geq 0$ with its Itô differential

$$
d x_{\Delta}(t)=f_{\Delta}\left(\bar{x}_{\Delta}(t), \bar{x}_{\Delta}(t-\tau)\right) d t+g_{\Delta}\left(\bar{x}_{\Delta}(t), \bar{x}_{\Delta}(t-\tau)\right) d B(t) .
$$

It is useful to know that $X_{\Delta}\left(t_{k}\right)=\bar{x}_{\Delta}\left(t_{k}\right)=x_{\Delta}\left(t_{k}\right)$ for every $k \geq-M$, namely they coincide at $t_{k}$. Of course, $\bar{x}_{\Delta}(t)$ is computable but $x_{\Delta}(t)$ is not in general. However, the following lemma shows that $x_{\Delta}(t)$ and $\bar{x}_{\Delta}(t)$ are close to each other in the sense of $L^{p}$. This indicates that it is sufficient to use $\bar{x}_{\Delta}(t)$ in practice. On the other hand, in our analysis, it is more convenient to work on both of them.

Lemma 2.5 For any $\Delta \in\left(0, \Delta^{*}\right]$ and any $p \geq 2$, we have

$$
\mathbb{E}\left|x_{\Delta}(t)-\bar{x}_{\Delta}(t)\right|^{p} \leq c_{p} \Delta^{p / 2}(h(\Delta))^{p}, \quad \forall t \geq 0,
$$

where $c_{p}$ is a positive constant dependent only on p. Consequently

$$
\lim _{\Delta \rightarrow 0} \mathbb{E}\left|x_{\Delta}(t)-\bar{x}_{\Delta}(t)\right|^{p}=0, \quad \forall t \geq 0 .
$$

Proof In what follows, we will use $c_{p}$ to stand for generic positive real constants dependent only on $p$ and its values may change between occurrences. Fix $\Delta \in\left(0, \Delta^{*}\right]$ arbitrarily. For any $t \geq 0$, there is a unique integer $k \geq 0$ such that $t_{k} \leq t<t_{k+1}$. By (2.8) as well as the Hölder inequality (see, e.g., [13, page 5]) and the moment property of the Itô integral (see, e.g., [13, Theorem 7.1 on page 39]), we can derive from (2.16) that

$$
\begin{aligned}
& \mathbb{E}\left|x_{\Delta}(t)-\bar{x}_{\Delta}(t)\right|^{p}=\mathbb{E}\left|x_{\Delta}(t)-x_{\Delta}\left(t_{k}\right)\right|^{p} \\
\leq & c_{p}\left(\mathbb{E}\left|\int_{t_{k}}^{t} f_{\Delta}\left(\bar{x}_{\Delta}(s), \bar{x}_{\Delta}(s-\tau)\right) d s\right|^{p}+\mathbb{E}\left|\int_{t_{k}}^{t} g_{\Delta}\left(\bar{x}_{\Delta}(s), \bar{x}_{\Delta}(s-\tau)\right) d B(s)\right|^{p}\right) \\
\leq & c_{p}\left(\Delta^{p-1} \mathbb{E} \int_{t_{k}}^{t}\left|f_{\Delta}\left(\bar{x}_{\Delta}(s), \bar{x}_{\Delta}(s-\tau)\right)\right|^{p} d s\right. \\
& \left.\quad+\Delta^{(p-2) / 2} \mathbb{E} \int_{t_{k}}^{t}\left|g_{\Delta}\left(\bar{x}_{\Delta}(s), \bar{x}_{\Delta}(s-\tau)\right)\right|^{p} d s\right) \\
\leq & c_{p} \Delta^{p / 2}(h(\Delta))^{p},
\end{aligned}
$$


which is (2.17). Noting from (2.6) that $\Delta^{p / 2}(h(\Delta))^{p} \leq \Delta^{p / 4}$, we obtain (2.18) from (2.17) immediately.

\section{Convergence in $L^{q}$ for $q \in[1,2)$}

From now on, we will fix $T>0$ arbitrarily. In this section, we will show that

$$
\lim _{\Delta \rightarrow 0} \mathbb{E}\left|x_{\Delta}(T)-x(T)\right|^{q}=0 \quad \text { and } \quad \lim _{\Delta \rightarrow 0} \mathbb{E}\left|\bar{x}_{\Delta}(T)-x(T)\right|^{q}=0
$$

for every $1 \leq q<2$. By (2.8), we see that for any given $\Delta \in\left(0, \Delta^{*}\right.$ ], both coefficients $f_{\Delta}$ and $g_{\Delta}$ are bounded. It therefore follows easily from (2.15) that for every $p \geq 2$,

$$
\mathbb{E}\left|x_{\Delta}(t)\right|^{p}<\infty, \quad \forall t \geq 0 .
$$

The following lemma gives an (uniformly) upper bound, independent of $\Delta$, for the second moment.

Lemma 3.1 Let Assumptions 2.1 and 2.2 hold. Then,

$$
\sup _{0<\Delta \leq \Delta^{*}} \sup _{0 \leq t \leq T} \mathbb{E}\left|x_{\Delta}(t)\right|^{2} \leq C,
$$

where, and from now on, $C$ stands for generic positive real constants dependent on $T, K_{1}, K_{2}, \xi$ (and $\bar{p}, K_{3}$ etc. as well in the next sections) but independent of $\Delta$ and its values may change between occurrences.

Proof Fix $\Delta \in\left(0, \Delta^{*}\right]$ and the initial data $\xi$ arbitrarily. By the Itô formula, we derive from (2.16) that for $0 \leq t \leq T$,

$$
\begin{aligned}
& \mathbb{E}\left|x_{\Delta}(t)\right|^{2}=|\xi(0)|^{2}+\mathbb{E} \int_{0}^{t}\left(2 x_{\Delta}^{T}(s) f_{\Delta}\left(\bar{x}_{\Delta}(s), \bar{x}_{\Delta}(s-\tau)\right)\right.\left.+\left|g_{\Delta}\left(\bar{x}_{\Delta}(s), \bar{x}_{\Delta}(s-\tau)\right)\right|^{2}\right) d s \\
&=|\xi(0)|^{2}+\mathbb{E} \int_{0}^{t}\left(2 \bar{x}_{\Delta}^{T}(s) f_{\Delta}\left(\bar{x}_{\Delta}(s), \bar{x}_{\Delta}(s-\tau)\right)\right. \\
&\left.+\left|g_{\Delta}\left(\bar{x}_{\Delta}(s), \bar{x}_{\Delta}(s-\tau)\right)\right|^{2}\right) d s \\
&+\mathbb{E} \int_{0}^{t} 2\left(x_{\Delta}(s)-\bar{x}_{\Delta}(s)\right)^{T} f_{\Delta}\left(\bar{x}_{\Delta}(s), \bar{x}_{\Delta}(s-\tau)\right) d s .
\end{aligned}
$$

By Lemma 2.4, we get

$$
\begin{aligned}
\mathbb{E}\left|x_{\Delta}(t)\right|^{2} \leq & |\xi(0)|^{2}+4 K_{1} \mathbb{E} \int_{0}^{t}\left(1+\left|\bar{x}_{\Delta}(s)\right|^{2}+\left|\bar{x}_{\Delta}(s-\tau)\right|^{2}\right) d s \\
& -2 K_{2} \mathbb{E} \int_{0}^{t}\left|\pi_{\Delta}\left(\bar{x}_{\Delta}(s)\right)\right|^{\beta} d s+2 K_{2} \mathbb{E} \int_{0}^{t}\left|\pi_{\Delta}\left(\bar{x}_{\Delta}(s-\tau)\right)\right|^{\beta} d s \\
& +2 \mathbb{E} \int_{0}^{t}\left|x_{\Delta}(s)-\bar{x}_{\Delta}(s)\right|\left|f_{\Delta}\left(\bar{x}_{\Delta}(s), \bar{x}_{\Delta}(s-\tau)\right)\right| d s .
\end{aligned}
$$


However,

$$
\begin{aligned}
& |\xi(0)|^{2}+4 K_{1} \mathbb{E} \int_{0}^{t}\left(1+\left|\bar{x}_{\Delta}(s)\right|^{2}+\left|\bar{x}_{\Delta}(s-\tau)\right|^{2}\right) d s \\
\leq & |\xi(0)|^{2}+4 K_{1} \int_{0}^{t}\left(1+\sup _{0 \leq u \leq s} \mathbb{E}\left|\bar{x}_{\Delta}(u)\right|^{2}+\sup _{0 \leq u \leq s} \mathbb{E}\left|\bar{x}_{\Delta}(u-\tau)\right|^{2}\right) d s \\
\leq & 4 K_{1} \int_{0}^{t}\left(1+\|\xi\|^{2}+2 \sup _{0 \leq u \leq s} \mathbb{E}\left|x_{\Delta}(u)\right|^{2}\right) d s \\
\leq & C+8 K_{1} \int_{0}^{t}\left(\sup _{0 \leq u \leq s} \mathbb{E}\left|x_{\Delta}(u)\right|^{2}\right) d s .
\end{aligned}
$$

Moreover,

$$
\begin{aligned}
& -2 K_{2} \mathbb{E} \int_{0}^{t}\left|\pi_{\Delta}\left(\bar{x}_{\Delta}(s)\right)\right|^{\beta} d s+2 K_{2} \mathbb{E} \int_{0}^{t}\left|\pi_{\Delta}\left(\bar{x}_{\Delta}(s-\tau)\right)\right|^{\beta} d s \\
= & -2 K_{2} \mathbb{E} \int_{0}^{t}\left|\pi_{\Delta}\left(\bar{x}_{\Delta}(s)\right)\right|^{\beta} d s+2 K_{2} \mathbb{E} \int_{-\tau}^{t-\tau}\left|\pi_{\Delta}\left(\bar{x}_{\Delta}(s)\right)\right|^{\beta} d s \\
\leq & 2 K_{2} \int_{-\tau}^{0}\left|\pi_{\Delta}\left(\bar{x}_{\Delta}(s)\right)\right|^{\beta} d s \leq 2 \tau K_{2}\|\xi\|^{\beta} .
\end{aligned}
$$

Furthermore, by Lemma 2.5 with $p=2$ and inequalities (2.8) and (2.6), we derive that

$$
\begin{aligned}
& \mathbb{E} \int_{0}^{t}\left|x_{\Delta}(s)-\bar{x}_{\Delta}(s)\right|\left|f_{\Delta}\left(\bar{x}_{\Delta}(s), \bar{x}_{\Delta}(s-\tau)\right)\right| d s \\
\leq & h(\Delta) \int_{0}^{T} \mathbb{E}\left|x_{\Delta}(s)-\bar{x}_{\Delta}(s)\right| d s \\
\leq & h(\Delta) \int_{0}^{T}\left(\mathbb{E}\left|x_{\Delta}(s)-\bar{x}_{\Delta}(s)\right|^{2}\right)^{1 / 2} d s \\
\leq & C(h(\Delta))^{2} \Delta^{1 / 2} \leq C .
\end{aligned}
$$

Substituting (3.3)-(3.5) into (3.2) yields

$$
\mathbb{E}\left|x_{\Delta}(t)\right|^{2} \leq C+8 K_{1} \int_{0}^{t}\left(\sup _{0 \leq u \leq s} \mathbb{E}\left|x_{\Delta}(u)\right|^{2}\right) d s .
$$

As this holds for any $t \in[0, T]$ while the sum of the right-hand-side (RHS) terms is non-decreasing in $t$, we then see

$$
\sup _{0 \leq u \leq t} \mathbb{E}\left|x_{\Delta}(u)\right|^{2} \leq C+8 K_{1} \int_{0}^{t}\left(\sup _{0 \leq u \leq s} \mathbb{E}\left|x_{\Delta}(u)\right|^{2}\right) d s .
$$

The well-known Gronwall inequality yields that

$$
\sup _{0 \leq u \leq T} \mathbb{E}\left|x_{\Delta}(u)\right|^{2} \leq C .
$$

As this holds for any $\Delta \in\left(0, \Delta^{*}\right]$ while $C$ is independent of $\Delta$, we obtain the required assertion (3.1). 
Let us present two more lemmas before we state one of our main results in this paper.

Lemma 3.2 Let Assumptions 2.1 and 2.2 hold. For any real number $R>\|\xi\|$, define the stopping time

$$
\tau_{R}=\inf \{t \geq 0:|x(t)| \geq R\}
$$

where throughout this paper, we set $\inf \emptyset=\infty$ (and as usual $\emptyset$ denotes the empty set). Then,

$$
\mathbb{P}\left(\tau_{R} \leq T\right) \leq \frac{C}{R^{2}} .
$$

(Recall that $C$ stands for generic positive real constants dependent on $T, K_{1}, K_{2}, \xi$ so $C$ here is independent of $R$.)

Proof By the Itô formula and Assumption 2.2, we derive that for $0 \leq t \leq T$,

$$
\begin{aligned}
\mathbb{E}\left|x\left(t \wedge \tau_{R}\right)\right|^{2} \leq & |\xi(0)|^{2}+2 K_{1} \mathbb{E} \int_{0}^{t \wedge \tau_{R}}\left(1+|x(s)|^{2}+|x(s-\tau)|^{2}\right) d s \\
& -2 K_{2} \mathbb{E} \int_{0}^{t \wedge \tau_{R}}|x(s)|^{\beta} d s+2 K_{2} \mathbb{E} \int_{0}^{t \wedge \tau_{R}}|x(s-\tau)|^{\beta} d s \\
\leq & |\xi(0)|^{2}+2 K_{1} T+2 K_{1} \mathbb{E} \int_{0}^{t}\left(\left|x\left(s \wedge \tau_{R}\right)\right|^{2}+\left|x\left((s-\tau) \wedge \tau_{R}\right)\right|^{2}\right) d s \\
& +2 K_{2} \int_{-\tau}^{0}|\xi(s)|^{\beta} d s \\
\leq & C+2 K_{1} \int_{0}^{t}\left(\mathbb{E}\left|x\left(s \wedge \tau_{R}\right)\right|^{2}+\mathbb{E}\left|x\left((s-\tau) \wedge \tau_{R}\right)\right|^{2}\right) d s \\
\leq & C+4 K_{1} \int_{0}^{t}\left(\sup _{0 \leq u \leq s} \mathbb{E}\left|x\left(u \wedge \tau_{R}\right)\right|^{2}\right) d s
\end{aligned}
$$

But the sum of the RHS terms is non-decreasing in $t$, we hence have

$$
\sup _{0 \leq u \leq t} \mathbb{E}\left|x\left(u \wedge \tau_{R}\right)\right|^{2} \leq C+4 K_{1} \int_{0}^{t}\left(\sup _{0 \leq u \leq s} \mathbb{E}\left|x\left(u \wedge \tau_{R}\right)\right|^{2}\right) d s .
$$

The Gronwall inequality shows

$$
\sup _{0 \leq u \leq T} \mathbb{E}\left|x\left(u \wedge \tau_{R}\right)\right|^{2} \leq C .
$$

In particular, we have

$$
\mathbb{E}\left|x\left(T \wedge \tau_{R}\right)\right|^{2} \leq C .
$$

This implies, by the Chebyshev inequality,

$$
R^{2} \mathbb{P}\left(\tau_{R} \leq T\right) \leq C
$$

and the assertion follows.

Lemma 3.3 Let Assumptions 2.1 and 2.2 hold. For any real number $R>\|\xi\|$ and $\Delta \in\left(0, \Delta^{*}\right]$, define the stopping time

$$
\rho_{\Delta, R}=\inf \left\{t \geq 0:\left|x_{\Delta}(t)\right| \geq R\right\} .
$$


Then

$$
\mathbb{P}\left(\rho_{\Delta, R} \leq T\right) \leq \frac{C}{R^{2}} .
$$

(Please recall that $C$ is independent of $\Delta$ and $R$.)

Proof We simply write $\rho_{\Delta, R}=\rho$. In the same way as (3.2) was obtained, we can show that for $0 \leq t \leq T$,

$$
\begin{aligned}
\mathbb{E}\left|x_{\Delta}(t \wedge \rho)\right|^{2} \leq & |\xi(0)|^{2}+4 K_{1} \mathbb{E} \int_{0}^{t \wedge \rho}\left(1+\left|\bar{x}_{\Delta}(s)\right|^{2}+\left|\bar{x}_{\Delta}(s-\tau)\right|^{2}\right) d s \\
& -2 K_{2} \mathbb{E} \int_{0}^{t \wedge \rho}\left|\pi_{\Delta}\left(\bar{x}_{\Delta}(s)\right)\right|^{\beta} d s+2 K_{2} \mathbb{E} \int_{0}^{t \wedge \rho}\left|\pi_{\Delta}\left(\bar{x}_{\Delta}(s-\tau)\right)\right|^{\beta} d s \\
& +2 \mathbb{E} \int_{0}^{t \wedge \rho}\left|x_{\Delta}(s)-\bar{x}_{\Delta}(s)\right|\left|f_{\Delta}\left(\bar{x}_{\Delta}(s), \bar{x}_{\Delta}(s-\tau)\right)\right| d s
\end{aligned}
$$

In the same way, as we performed in the proofs of Lemmas 3.1 and 3.2, we can then show that

$$
\begin{aligned}
\mathbb{E}\left|x_{\Delta}(t \wedge \rho)\right|^{2} \leq & C+8 K_{1} \int_{0}^{t}\left(\sup _{0 \leq u \leq s} \mathbb{E}\left|\bar{x}_{\Delta}(u \wedge \rho)\right|^{2}\right) d s \\
& +2 \mathbb{E} \int_{0}^{t}\left|x_{\Delta}(s)-\bar{x}_{\Delta}(s)\right|\left|f_{\Delta}\left(\bar{x}_{\Delta}(s), \bar{x}_{\Delta}(s-\tau)\right)\right| d s .
\end{aligned}
$$

This, together with (3.5), implies

$$
\mathbb{E}\left|x_{\Delta}(t \wedge \rho)\right|^{2} \leq C+8 K_{1} \int_{0}^{t}\left(\sup _{0 \leq u \leq s} \mathbb{E}\left|\bar{x}_{\Delta}(u \wedge \rho)\right|^{2}\right) d s .
$$

Noting that the sum of the RHS terms is increasing in $t$ while

$$
\sup _{0 \leq u \leq s} \mathbb{E}\left|\bar{x}_{\Delta}(u \wedge \rho)\right|^{2} \leq \sup _{0 \leq u \leq s} \mathbb{E}\left|x_{\Delta}(u \wedge \rho)\right|^{2},
$$

we get

$$
\sup _{0 \leq u \leq t} \mathbb{E}\left|x_{\Delta}(u \wedge \rho)\right|^{2} \leq C+8 K_{1} \int_{0}^{t}\left(\sup _{0 \leq u \leq s} \mathbb{E}\left|x_{\Delta}(u \wedge \rho)\right|^{2}\right) d s .
$$

The Gronwall inequality shows

$$
\sup _{0 \leq u \leq T} \mathbb{E}\left|x_{\Delta}(u \wedge \rho)\right|^{2} \leq C .
$$

This implies the required assertion (3.7) easily.

For the numerical solutions to converge to the true solution in $L^{q}$, we need to assume that the initial data are Hölder continuous with exponent $\gamma$ (or $\gamma$-Hölder continuous). This is a standard condition which is also needed for the classical EM method under the global Lipschitz condition (see, e.g., [18, 22]).

Assumption 3.4 There is a pair of constants $K_{3}>0$ and $\gamma \in(0,1]$ such that the initial data $\xi$ satisfies

$$
|\xi(u)-\xi(v)| \leq K_{3}|u-v|^{\gamma}, \quad-\tau \leq v<u \leq 0 .
$$


We can now show one of our main results in this paper.

Theorem 3.5 Let Assumptions 2.1, 2.2 and 3.4 hold. Then, for any $q \in[1,2)$,

$$
\lim _{\Delta \rightarrow 0} \mathbb{E}\left|x_{\Delta}(T)-x(T)\right|^{q}=0 \quad \text { and } \quad \lim _{\Delta \rightarrow 0} \mathbb{E}\left|\bar{x}_{\Delta}(T)-x(T)\right|^{q}=0 .
$$

Proof Let $\tau_{R}$ and $\rho_{\Delta, R}$ be the same as before. Set

$$
\theta_{\Delta, R}=\tau_{R} \wedge \rho_{\Delta, R} \text { and } e_{\Delta}(T)=x_{\Delta}(T)-x(T) .
$$

Obviously,

$$
\mathbb{E}\left|e_{\Delta}(T)\right|^{q}=\mathbb{E}\left(\left|e_{\Delta}(T)\right|^{q} I_{\left\{\theta_{\Delta, R}>T\right\}}\right)+\mathbb{E}\left(\left|e_{\Delta}(T)\right|^{q} I_{\left\{\theta_{\Delta, R} \leq T\right\}}\right) .
$$

Let $\delta>0$ be arbitrary. Using the Young inequality

$$
u^{\alpha} v^{(1-\alpha)} \leq \alpha u+(1-\alpha) v \quad \forall u, v>0, \alpha \in(0,1),
$$

we see that

$$
a^{q} b=\left(\delta a^{2}\right)^{q / 2}\left(\frac{b^{2 /(2-q)}}{\delta^{q /(2-q)}}\right)^{(2-q) / 2} \leq \frac{q \delta}{2} a^{2}+\frac{2-q}{2 \delta^{q /(2-q)}} b^{2 /(2-q)}, \quad \forall a, b>0,
$$

and hence have

$$
\mathbb{E}\left(\left|e_{\Delta}(T)\right|^{q} I_{\left\{\theta_{\Delta, R} \leq T\right\}}\right) \leq \frac{q \delta}{2} \mathbb{E}\left|e_{\Delta}(T)\right|^{2}+\frac{2-q}{2 \delta^{q /(2-q)}} \mathbb{P}\left(\theta_{\Delta, R} \leq T\right) .
$$

By Lemmas 2.3 and 3.1, we have

$$
\mathbb{E}\left|e_{\Delta}(T)\right|^{2} \leq C
$$

while by Lemmas 3.2 and 3.3,

$$
\mathbb{P}\left(\theta_{\Delta, R} \leq T\right) \leq \mathbb{P}\left(\tau_{R} \leq T\right)+\mathbb{P}\left(\rho_{\Delta, R} \leq T\right) \leq \frac{C}{R^{2}}
$$

We hence have

$$
\mathbb{E}\left(\left|e_{\Delta}(T)\right|^{q} I_{\left\{\theta_{\Delta, R} \leq T\right\}}\right) \leq \frac{C q \delta}{2}+\frac{C(2-q)}{2 R^{2} \delta^{q /(2-q)}} .
$$

Substituting this into (3.11) yields

$$
\mathbb{E}\left|e_{\Delta}(T)\right|^{q} \leq \mathbb{E}\left(\left|e_{\Delta}(T)\right|^{q} I_{\left\{\theta_{\Delta, R}>T\right\}}\right)+\frac{C q \delta}{2}+\frac{C(2-q)}{2 R^{2} \delta^{q /(2-q)}} .
$$

Now, let $\varepsilon>0$ be arbitrary. Choose $\delta$ sufficiently small for $C q \delta / 2 \leq \varepsilon / 3$ and then choose $R$ sufficiently large for

$$
\frac{C(2-q)}{2 R^{2} \delta^{q /(2-q)}} \leq \frac{\varepsilon}{3} .
$$

We then see from (3.13) that for this particularly chosen $R$,

$$
\mathbb{E}\left|e_{\Delta}(T)\right|^{q} \leq \mathbb{E}\left(\left|e_{\Delta}(T)\right|^{q} I_{\left\{\theta_{\Delta, R}>T\right\}}\right)+\frac{2 \varepsilon}{3} .
$$

If we can show that for all sufficiently small $\Delta$,

$$
\mathbb{E}\left(\left|e_{\Delta}(T)\right|^{q} I_{\left\{\theta_{\Delta, R}>T\right\}}\right) \leq \frac{\varepsilon}{3},
$$


we have

$$
\lim _{\Delta \rightarrow 0} \mathbb{E}\left|e_{\Delta}(T)\right|^{q}=0,
$$

and then by Lemma 2.5, we also have

$$
\lim _{\Delta \rightarrow 0} \mathbb{E}\left|x(T)-\bar{x}_{\Delta}(T)\right|^{q}=0 .
$$

In other words, to complete our proof, all we need is to show (3.15). For this purpose, we define the truncated functions

$$
\begin{aligned}
& F_{R}(x, y)=f\left((|x| \wedge R) \frac{x}{|x|},(|y| \wedge R) \frac{y}{|y|}\right) \text { and } \\
& G_{R}(x, y)=g\left((|x| \wedge R) \frac{x}{|x|},(|y| \wedge R) \frac{y}{|y|}\right)
\end{aligned}
$$

for $x, y \in \mathbb{R}^{n}$. Without loss of any generality, we may assume that $\Delta^{*}$ is already sufficiently small for $\mu^{-1}\left(h\left(\Delta^{*}\right)\right) \geq R$. Hence, for all $\Delta \in\left(0, \Delta^{*}\right]$, we have that

$$
f_{\Delta}(x, y)=F_{R}(x, y) \quad \text { and } \quad g_{\Delta}(x, y)=G_{R}(x, y)
$$

for those $x, y \in \mathbb{R}^{n}$ with $|x| \vee|y| \leq R$. Consider the SDDE

$$
d z(t)=F_{R}(z(t), z(t-\tau)) d t+G_{R}(z(t), z(t-\tau)) d B(t)
$$

on $t \geq 0$ with the initial data $z(u)=\xi(u)$ on $u \in[-\tau, 0]$. By Assumption 2.1, we see that both $F_{R}(x, y)$ and $G_{R}(x, y)$ are globally Lipschitz continuous with the Lipschitz constant $K_{R}$. So the $\operatorname{SDDE}$ (3.16) has a unique global solution $z(t)$ on $t \geq-\tau$. It is straightforward to see that

$$
\mathbb{P}\left\{x\left(t \wedge \tau_{R}\right)=z\left(t \wedge \tau_{R}\right) \text { for all } 0 \leq t \leq T\right\}=1 .
$$

On the other hand, for each step size $\Delta \in\left(0, \Delta^{*}\right]$, we can apply the (classical) EM method to the SDDE (3.16) and we denote by $z_{\Delta}(t)$ the continuous-time continuous EM solution. It is again straightforward to see that

$$
\mathbb{P}\left\{x_{\Delta}\left(t \wedge \rho_{\Delta, R}\right)=z_{\Delta}\left(t \wedge \rho_{\Delta, R}\right) \text { for all } 0 \leq t \leq T\right\}=1 .
$$

However, it is well known (see, e.g., [18]) that

$$
\mathbb{E}\left(\sup _{0 \leq t \leq T}\left|z(t)-z_{\Delta}(t)\right|^{q}\right) \leq H \Delta^{q(0.5 \wedge \gamma)},
$$

where $H$ is a positive constant dependent on $K_{R}, T, \xi, q$ but independent of $\Delta$. Consequently,

$$
\mathbb{E}\left(\sup _{0 \leq t \leq T}\left|z\left(t \wedge \theta_{\Delta, R}\right)-z_{\Delta}\left(t \wedge \theta_{\Delta, R}\right)\right|^{q}\right) \leq H \Delta^{q(0.5 \wedge \gamma)} .
$$


Using (3.17) and (3.18), we then have

$$
\mathbb{E}\left(\sup _{0 \leq t \leq T}\left|x\left(t \wedge \theta_{\Delta, R}\right)-x_{\Delta}\left(t \wedge \theta_{\Delta, R}\right)\right|^{q}\right) \leq H \Delta^{q(0.5 \wedge \gamma)},
$$

which implies

$$
\mathbb{E}\left(\left|x\left(T \wedge \theta_{\Delta, R}\right)-x_{\Delta}\left(T \wedge \theta_{\Delta, R}\right)\right|^{q}\right) \leq H \Delta^{q(0.5 \wedge \gamma)} .
$$

Finally,

$$
\begin{aligned}
\mathbb{E}\left(\left|e_{\Delta}(T)\right|^{q} I_{\left\{\theta_{\Delta, R}>T\right\}}\right) & =\mathbb{E}\left(\left|e_{\Delta}\left(T \wedge \theta_{\Delta, R}\right)\right|^{q} I_{\left\{\theta_{\Delta, R}>T\right\}}\right) \\
& \leq \mathbb{E}\left(\left|x\left(T \wedge \theta_{\Delta, R}\right)-x_{\Delta}\left(T \wedge \theta_{\Delta, R}\right)\right|^{q}\right) \\
& \leq H \Delta^{q(0.5 \wedge \gamma)} .
\end{aligned}
$$

This implies (3.15) as desired. The proof is therefore complete.

Lets make a useful remark which will be used in next sections before we discuss an example to illustrate our theory.

Remark 3.6 It is known (see, e.g., [18]) that (3.19) holds for any $q \geq 2$. We hence see from the proof above that both (3.20) and (3.21) hold for any $q \geq 2$ too.

Example 3.7 Consider a two-dimensional SDDE

$\left\{\begin{array}{l}d x_{1}(t)=x_{1}(t)\left(\left[a_{11}+a_{12} x_{2}(t-\tau)-a_{13} x_{1}^{2}(t)\right] d t+\left[a_{14} x_{1}(t)+a_{15} x_{2}(t-\tau)\right] d B(t)\right), \\ d x_{2}(t)=x_{2}(t)\left(\left[a_{21}+a_{22} x_{1}(t-\tau)-a_{23} x_{2}^{2}(t)\right] d t+\left[a_{24} x_{1}(t)+a_{25} x_{1}(t-\tau)\right] d B(t)\right),\end{array}\right.$

on $t \geq 0$ with the initial data $\left\{\left(x_{1}(\theta), x_{2}(\theta)\right)^{T}:-\tau \leq \theta \leq 0\right\}=\xi \epsilon$ $C\left([-\tau, 0] ;(0, \infty)^{2}\right)$, where $B(t)$ is a scalar Brownian motion and $a_{i j}(i=1,2$ and $1 \leq j \leq 5)$ are all positive numbers. Let $a_{j}=a_{1 j} \vee a_{2 j}$ for $j=1,2,4,5$ and $a_{3}=a_{13} \wedge a_{23}$ and assume that

$$
a_{3}>a_{4}^{2}+1.5 a_{5}^{2} .
$$

This is an SDDE population model for two species (see, e.g., [2]). Our method can be applied to a more general SDDE model for multiple species. We only consider the two species case here in order to avoid the notation becoming too complicated but our method is illustrated fully. It is known (see, e.g., [2]) that given the initial data $\xi \in C\left([-\tau, 0] ;(0, \infty)^{2}\right)$, the solution will remain positive for all $t \geq 0$ with probability 1 . We can therefore regard (3.22) as an SDDE in $\mathbb{R}^{2}$ with the coefficients

$$
\begin{aligned}
& f(x, y)=\left(\begin{array}{l}
x_{1}\left(a_{11}+a_{12} y_{2}-a_{13} x_{1}^{2}\right) \\
x_{2}\left(a_{21}+a_{22} y_{1}-a_{23} x_{2}^{2}\right)
\end{array}\right) \text { and } \\
& g(x, y)=\left(\begin{array}{l}
a_{14} x_{1}+a_{15} y_{2} \\
a_{24} x_{2}+a_{25} y_{1}
\end{array}\right) \text { for } x, y \in \mathbb{R}^{2} .
\end{aligned}
$$


It is obvious that these coefficients are locally Lipschitz continuous, namely, they satisfy Assumption 2.1. We also assume that the initial data satisfy Assumption 3.4. Moreover, let $\delta=a_{3}-a_{4}^{2}-1.5 a_{5}^{2}$, which is positive by (3.23). We then derive

$$
\begin{aligned}
& x^{T} f(x, y)+\frac{1}{2}|g(x, y)|^{2} \\
= & a_{11} x_{1}^{2}+a_{12} x_{1}^{2} y_{2}-a_{13} x_{1}^{4}+a_{14}^{2} x_{1}^{4}+a_{15}^{2} x_{1}^{2} y_{2}^{2} \\
& +a_{21} x_{2}^{2}+a_{22} x_{2}^{2} y_{1}-a_{23} x_{2}^{4}+a_{24}^{2} x_{2}^{4}+a_{25}^{2} x_{2}^{2} y_{1}^{2} \\
\leq & a_{1} x^{2}+\left(a_{2}^{2} / 4 \delta\right) y^{2}-\left(a_{3}-\delta-a_{4}^{2}-0.5 a_{5}^{2}\right)\left(x_{1}^{4}+x_{2}^{4}\right)+0.5 a_{5}^{2}\left(y_{1}^{4}+y_{2}^{4}\right) \\
\leq & a_{1} x^{2}+\left(a_{2}^{2} / 4 \delta\right) y^{2}-0.5\left(a_{3}-\delta-a_{4}^{2}-0.5 a_{5}^{2}\right)|x|^{4}+0.5 a_{5}^{2}|y|^{4} \\
\leq & \left(a_{1} \vee\left(a_{2}^{2} / 4 \delta\right)\right)\left(1+x^{2}+y^{2}\right)-0.5 a_{5}^{2} x^{4}+0.5 a_{5}^{2} y^{4} .
\end{aligned}
$$

That is, Assumption 2.2 is satisfied as well. We can therefore apply the truncated EM method to obtain the numerical solutions of the SDDE (3.22). For this purpose, we observe that, for $r \geq 1$,

$$
\sup _{|x| \vee|y| \leq r}(|f(x, y)| \vee|g(x, y)|) \leq 2\left[\left(a_{1} r+a_{2} r^{2}+\hat{a}_{3} r^{3}\right) \vee\left(\left(a_{4}+a_{5}\right) r^{2}\right)\right] \leq a r^{3},
$$

where $\hat{a}_{3}=a_{13} \vee a_{23}$ and $a=2\left[\left(a_{1}+a_{2}+\hat{a}_{3}\right) \vee\left(a_{4}+a_{5}\right)\right]$. We can therefore define $\mu: \mathbb{R}_{+} \rightarrow \mathbb{R}_{+}$by

$$
\mu(r)=a r^{3}, \quad r \geq 0 .
$$

Its inverse function $\mu^{-1}: \mathbb{R}_{+} \rightarrow \mathbb{R}_{+}$has the form

$$
\mu^{-1}(r)=\left(\frac{r}{a}\right)^{1 / 3}, \quad r \geq 0 .
$$

Let $\rho \in(0,1 / 4]$ and $\Delta^{*}=(1 \vee(8 a))^{-1 / \rho} \in(0,1]$. Define $h(\Delta)=\Delta^{-\rho}$ for $\Delta \in\left(0, \Delta^{*}\right]$. We then see that $h\left(\Delta^{*}\right) \geq 8 a=\mu(2), \lim _{\Delta \rightarrow 0} h(\Delta)=\infty$ and

$$
\Delta^{1 / 4} h(\Delta)=\Delta^{1 / 4-\rho} \leq 1, \quad \forall \Delta \in\left(0, \Delta^{*}\right]
$$

as required by (2.6). With these chosen functions $\mu$ and $h$, we can then apply the truncated EM method to obtain the numerical solutions $x_{\Delta}(t)$ and $\bar{x}_{\Delta}(t)$ of the SDDE (3.22). Moreover, Theorem 3.5 shows that these numerical solutions will converge to the true solution $x(t)$ in the sense that

$$
\lim _{\Delta \rightarrow 0} \mathbb{E}\left|x_{\Delta}(t)-x(t)\right|^{q}=0 \quad \text { and } \quad \lim _{\Delta \rightarrow 0} \mathbb{E}\left|\bar{x}_{\Delta}(t)-x(t)\right|^{q}=0
$$

for any $q \in[1,2)$.

\section{Convergence in $L^{q}$ for $q \geq 2$}

In the previous section, we showed that the truncated EM solutions $x_{\Delta}(T)$ and $\bar{x}_{\Delta}(T)$ will converge to the true solution $x(T)$ in $L^{q}$ for any $q \in[1,2)$. This is sufficient for some applications, for example, when we need to approximate the mean value of the solution or the European call option value (see, e.g., [10]). However, we sometimes 
need to approximate the variance or higher moment of the solution. In these situations, we need to have the convergence in $L^{q}$ for $q \geq 2$. For this purpose, we impose a stronger Khasminskii-type condition.

Assumption 4.1 There is a pair of constants $\bar{p}>2$ and $K_{1}>0$ such that

$$
x^{T} f(x, y)+\frac{\bar{p}-1}{2}|g(x, y)|^{2} \leq K_{1}\left(1+|x|^{2}+|y|^{2}\right)
$$

for all $(x, y) \in \mathbb{R}^{n} \times \mathbb{R}^{n}$.

Once again, the truncated functions $f_{\Delta}$ and $g_{\Delta}$ preserve this condition nicely.

Lemma 4.2 Let Assumption 4.1 hold. Then, for every $\Delta \in\left(0, \Delta^{*}\right]$, we have

$$
x^{T} f_{\Delta}(x, y)+\frac{\bar{p}-1}{2}\left|g_{\Delta}(x, y)\right|^{2} \leq 2 K_{1}\left(1+|x|^{2}+|y|^{2}\right)
$$

for all $x, y \in \mathbb{R}^{n}$.

This lemma can be proved in the same way as Lemma 2.4 was proved. We also cite a stronger result than Lemma 2.3 from [17].

Lemma 4.3 Let Assumptions 2.1 and 4.1 hold. Then for any given initial data (2.2), there is a unique global solution $x(t)$ to (2.1) on $t \in[-\tau, \infty)$. Moreover, the solution has the property that

$$
\sup _{-\tau \leq t \leq T} \mathbb{E}|x(t)|^{\bar{p}}<\infty .
$$

Let us now establish a stronger result than Lemma 3.1.

Lemma 4.4 Let Assumptions 2.1 and 4.1 hold. Then,

$$
\sup _{0<\Delta \leq \Delta^{*}} \sup _{0 \leq t \leq T} \mathbb{E}\left|x_{\Delta}(t)\right|^{\bar{p}} \leq C .
$$

Proof Fix any $\Delta \in\left(0, \Delta^{*}\right]$. By the Itô formula, we derive from (2.16) that, for $0 \leq t \leq T$

$$
\begin{aligned}
\mathbb{E}\left|x_{\Delta}(t)\right|^{\bar{p}} \leq & |\xi(0)|^{\bar{p}}+\mathbb{E} \int_{0}^{t} \bar{p}\left|x_{\Delta}(s)\right|^{\bar{p}-2} \\
& \times\left(x_{\Delta}^{T}(s) f_{\Delta}\left(\bar{x}_{\Delta}(s), \bar{x}_{\Delta}(s-\tau)\right)+\frac{\bar{p}-1}{2}\left|g_{\Delta}\left(\bar{x}_{\Delta}(s), \bar{x}_{\Delta}(s-\tau)\right)\right|^{2}\right) d s \\
= & |\xi(0)|^{\bar{p}}+\mathbb{E} \int_{0}^{t} \bar{p}\left|x_{\Delta}(s)\right|^{\bar{p}-2} \\
& \times\left(\bar{x}_{\Delta}^{T}(s) f_{\Delta}\left(\bar{x}_{\Delta}(s), \bar{x}_{\Delta}(s-\tau)\right)+\frac{\bar{p}-1}{2}\left|g_{\Delta}\left(\bar{x}_{\Delta}(s), \bar{x}_{\Delta}(s-\tau)\right)\right|^{2}\right) d s \\
& +\mathbb{E} \int_{0}^{t} \bar{p}\left|x_{\Delta}(s)\right|^{\bar{p}-2}\left(x_{\Delta}(s)-\bar{x}_{\Delta}(s)\right)^{T} f_{\Delta}\left(\bar{x}_{\Delta}(s), \bar{x}_{\Delta}(s-\tau)\right) d s .
\end{aligned}
$$


Noting from the Young inequality (3.12) that

$$
a^{\bar{p}-2} b \leq \frac{\bar{p}-2}{\bar{p}} a^{\bar{p}}+\frac{2}{\bar{p}} b^{\bar{p} / 2}, \quad \forall a, b \geq 0,
$$

as well as using Lemma 4.2, we then have

$$
\begin{aligned}
\mathbb{E}\left|x_{\Delta}(t)\right|^{\bar{p}} \leq & |\xi(0)|^{\bar{p}}+\mathbb{E} \int_{0}^{t} 2 \bar{p} K_{1}\left|x_{\Delta}(s)\right|^{\bar{p}-2}\left(1+\left|\bar{x}_{\Delta}(s)\right|^{2}+\left|\bar{x}_{\Delta}(s-\tau)\right|^{2}\right) d s \\
& +(\bar{p}-2) \mathbb{E} \int_{0}^{t}\left|x_{\Delta}(s)\right|^{\bar{p}} d s \\
& +2 \mathbb{E} \int_{0}^{t}\left|x_{\Delta}(s)-\bar{x}_{\Delta}(s)\right|^{\bar{p} / 2}\left|f_{\Delta}\left(\bar{x}_{\Delta}(s), \bar{x}_{\Delta}(s-\tau)\right)\right|^{\bar{p} / 2} d s \\
\leq & C+C \int_{0}^{t}\left(\mathbb{E}\left|x_{\Delta}(s)\right|^{\bar{p}}+\mathbb{E}\left|\bar{x}_{\Delta}(s)\right|^{\bar{p}}+\mathbb{E}\left|\bar{x}_{\Delta}(s-\tau)\right|^{\bar{p}}\right) d s \\
& +2 \mathbb{E} \int_{0}^{T}\left|x_{\Delta}(s)-\bar{x}_{\Delta}(s)\right|^{\bar{p} / 2}\left|f_{\Delta}\left(\bar{x}_{\Delta}(s), \bar{x}_{\Delta}(s-\tau)\right)\right|^{\bar{p} / 2} d s .
\end{aligned}
$$

But, by Lemma 2.5 with $p=\bar{p}$ and inequalities (2.8) and (2.6), we have

$$
\begin{aligned}
& \mathbb{E} \int_{0}^{T}\left|x_{\Delta}(s)-\bar{x}_{\Delta}(s)\right|^{\bar{p} / 2}\left|f_{\Delta}\left(\bar{x}_{\Delta}(s), \bar{x}_{\Delta}(s-\tau)\right)\right|^{\bar{p} / 2} d s \\
\leq & (h(\Delta))^{\bar{p} / 2} \int_{0}^{T} \mathbb{E}\left(\left|x_{\Delta}(s)-\bar{x}_{\Delta}(s)\right|^{\bar{p} / 2}\right) d s \\
\leq & (h(\Delta))^{\bar{p} / 2} \int_{0}^{T}\left(\mathbb{E}\left|x_{\Delta}(s)-\bar{x}_{\Delta}(s)\right|^{\bar{p}}\right)^{1 / 2} d s \\
\leq & c_{\bar{p}} T(h(\Delta))^{\bar{p}} \Delta^{\bar{p} / 4} \leq c_{\bar{p}} T .
\end{aligned}
$$

We therefore have

$$
\begin{aligned}
\mathbb{E}\left|x_{\Delta}(t)\right|^{\bar{p}} & \leq C+C \int_{0}^{t}\left(\mathbb{E}\left|x_{\Delta}(s)\right|^{\bar{p}}+\mathbb{E}\left|\bar{x}_{\Delta}(s)\right|^{\bar{p}}+\mathbb{E}\left|\bar{x}_{\Delta}(s-\tau)\right|^{\bar{p}}\right) d s \\
& \leq C+C \int_{0}^{t}\left(\sup _{0 \leq u \leq s} \mathbb{E}\left|x_{\Delta}(u)\right|^{\bar{p}}\right) d s .
\end{aligned}
$$

As this holds for any $t \in[0, T]$ while the sum of the RHS terms is non-decreasing in $t$, we then see

$$
\sup _{0 \leq u \leq t} \mathbb{E}\left|x_{\Delta}(u)\right|^{\bar{p}} \leq C+C \int_{0}^{t}\left(\sup _{0 \leq u \leq s} \mathbb{E}\left|x_{\Delta}(u)\right|^{\bar{p}}\right) d s .
$$

The well-known Gronwall inequality yields that

$$
\sup _{0 \leq u \leq T} \mathbb{E}\left|x_{\Delta}(u)\right|^{\bar{p}} \leq C .
$$

As this holds for any $\Delta \in\left(0, \Delta^{*}\right]$ while $C$ is independent of $\Delta$, we see the required assertion (4.4).

The following two lemmas are the analogues of Lemmas 3.2 and 3.3. 
Lemma 4.5 Let Assumptions 2.1 and 4.1 hold. For any real number $R>\|\xi\|$, define the stopping time $\tau_{R}=\inf \{t \geq 0:|x(t)| \geq R\}$. Then

$$
\mathbb{P}\left(\tau_{R} \leq T\right) \leq \frac{C}{R^{\bar{p}}}
$$

Lemma 4.6 Let Assumptions 2.1 and 4.1 hold. For any real number $R>\|\xi\|$ and $\Delta \in\left(0, \Delta^{*}\right]$, define the stopping time $\rho_{\Delta, R}=\inf \left\{t \geq 0:\left|x_{\Delta}(t)\right| \geq R\right\}$. Then

$$
\mathbb{P}\left(\rho_{\Delta, R} \leq T\right) \leq \frac{C}{R^{\bar{p}}}
$$

Their proofs are similar to those of Lemmas 3.2 and 3.3, respectively, so are omitted. We can now state our main result in this section.

Theorem 4.7 Let Assumptions 2.1, 3.4 and 4.1 hold. Then, for any $q \in[2, \bar{p})$,

$$
\lim _{\Delta \rightarrow 0} \mathbb{E}\left|x_{\Delta}(T)-x(T)\right|^{q}=0 \quad \text { and } \quad \lim _{\Delta \rightarrow 0} \mathbb{E}\left|\bar{x}_{\Delta}(T)-x(T)\right|^{q}=0 .
$$

Proof We use the same notation as in the proof of Theorem 3.5. Fix any $q \in[2, \bar{p})$. Using the Young inequality (3.12), we can show that for any $\delta>0$,

$$
\mathbb{E}\left|e_{\Delta}(T)\right|^{q} \leq \mathbb{E}\left(\left|e_{\Delta}(T)\right|^{q} I_{\left\{\theta_{\Delta, R}>T\right\}}\right)+\frac{q \delta}{\bar{p}} \mathbb{E}\left|e_{\Delta}(T)\right|^{\bar{p}}+\frac{\bar{p}-q}{\bar{p} \delta^{q /(\bar{p}-q)}} \mathbb{P}\left(\theta_{\Delta, R} \leq T\right) .
$$

By Lemmas 4.3 and 4.4, we have

$$
\mathbb{E}\left|e_{\Delta}(T)\right|^{\bar{p}} \leq C
$$

while by Lemmas 4.5 and 4.6,

$$
\mathbb{P}\left(\theta_{\Delta, R} \leq T\right) \leq \mathbb{P}\left(\tau_{R} \leq T\right)+\mathbb{P}\left(\rho_{\Delta, R} \leq T\right) \leq \frac{C}{R^{\bar{p}}} .
$$

Using these and (3.21) (please recall Remark 3.6), we obtain

$$
\mathbb{E}\left|e_{\Delta}(T)\right|^{q} \leq H \Delta^{q(0.5 \wedge \gamma)}+\frac{C q \delta}{\bar{p}}+\frac{C(\bar{p}-q)}{\bar{p} R^{\bar{p}} \delta^{q /(\bar{p}-q)}} .
$$

Now, for any $\varepsilon>0$, we first choose $\delta$ sufficiently small for $C q \delta / \bar{p} \leq \varepsilon / 3$ and then choose $R$ sufficiently large for

$$
\frac{C(\bar{p}-q)}{\bar{p} R^{\bar{p}} \delta^{q /(\bar{p}-q)}} \leq \frac{\varepsilon}{3},
$$

and further then choose $\Delta$ sufficiently small for $H \Delta^{q(0.5 \wedge \gamma)} \leq \varepsilon / 3$ to get that

$$
\mathbb{E}\left|e_{\Delta}(T)\right|^{q} \leq \varepsilon
$$

In other words, we have shown that

$$
\lim _{\Delta \rightarrow 0} \mathbb{E}\left|e_{\Delta}(T)\right|^{q}=0 .
$$


This, along with Lemma 2.5, implies another assertion

$$
\lim _{\Delta \rightarrow 0} \mathbb{E}\left|x(T)-\bar{x}_{\Delta}(T)\right|^{q}=0 .
$$

The proof is therefore complete.

Let us now discuss an example to illustrate this theorem before we study the convergence rates.

Example 4.8 Consider the scalar SDDE

$$
d x(t)=f(x(t), x(t-\tau)) d t+g(x(t), x(t-\tau)) d B(t), \quad t \geq 0,
$$

with the initial data $\{x(\theta):-\tau \leq \theta \leq 0\}=\xi \in C([-\tau, 0] ; \mathbb{R})$ which satisfy Assumption 3.4, where

$$
f(x, y)=a_{1}+a_{2}|y|^{4 / 3}-a_{3} x^{3} \quad \text { and } \quad g(x, y)=a_{4}|x|^{3 / 2}+a_{5} y, \quad x, y \in \mathbb{R},
$$

and $a_{1}, \cdots, a_{5}$ are all real numbers with $a_{3}>0$. Clearly, the coefficients $f$ and $g$ are locally Lipschitz continuous, namely, they satisfy Assumption 2.1. Moreover, for any $\bar{p}>2$, we have

$$
\begin{aligned}
x f(x, y)+\frac{\bar{p}-1}{2}|g(x, y)|^{2} \leq & \left|a_{1}\right||x|+\left|a_{2}\right||x||y|^{4 / 3}-a_{3}|x|^{4} \\
& +(\bar{p}-1)\left(\left|a_{4}\right||x|^{3}+\left|a_{5}\right||y|^{2}\right) .
\end{aligned}
$$

But, by the Young inequality (3.12),

$$
|x||y|^{4 / 3}=\left(|x|^{3}\right)^{1 / 3}\left(|y|^{2}\right)^{2 / 3} \leq|x|^{3}+|y|^{2} .
$$

We therefore have

$$
\begin{aligned}
& x f(x, y)+\frac{\bar{p}-1}{2}|g(x, y)|^{2} \\
\leq & \left|a_{1}\right||x|+\left(\left|a_{2}\right|+\left|a_{4}\right|(\bar{p}-1)\right)|x|^{3}-a_{3}|x|^{4}+\left(\left|a_{2}\right|+a_{5}(\bar{p}-1)\right)|y|^{2} \\
\leq & K_{1}\left(1+|y|^{2}\right),
\end{aligned}
$$

where $K_{1}=\left(\left|a_{2}\right|+\left|a_{5}\right|(\bar{p}-1)\right) \vee K$ and

$$
K=\sup _{u \geq 0}\left[\left|a_{1}\right| u+\left(\left|a_{2}\right|+\left|a_{4}\right|(\bar{p}-1)\right) u^{3}-a_{3} u^{4}\right]<\infty .
$$

That is, Assumption 4.1 is satisfied for any $\bar{p}>2$. To apply Theorem 4.7 , we still need to design functions $\mu$ and $h$ satisfying (2.5) and (2.6). Note that

$$
\sup _{|x| \leq u}(|f(x)| \vee|g(x)|) \leq \hat{a} u^{3}, \quad \forall u \geq 1,
$$

where $\hat{a}=\left(\left|a_{1}\right|+\left|a_{2}\right|+a_{3}\right) \vee\left(\left|a_{4}\right|+\left|a_{5}\right|\right)$. We can hence have $\mu(u)=\hat{a} u^{3}$ and its inverse function $\mu^{-1}(u)=(u / \hat{a})^{1 / 3}$ for $u \geq 0$. For $\varepsilon \in(0,1 / 4]$, we define $h(\Delta)=\Delta^{-\varepsilon}$ for $\Delta>0$. Letting $\Delta^{*} \in(0,1]$ be sufficiently small, we can make (2.6) hold. By Theorem 4.7, we can then conclude that the truncated EM solutions will converge to the true solution $x(t)$ in the sense that

$$
\lim _{\Delta \rightarrow 0} \mathbb{E}\left|x_{\Delta}(T)-x(T)\right|^{q}=0 \quad \text { and } \quad \lim _{\Delta \rightarrow 0} \mathbb{E}\left|\bar{x}_{\Delta}(T)-x(T)\right|^{q}=0
$$

for every $q \geq 2$. 


\section{Convergence rates}

In the previous sections, we showed the convergence in $L^{q}$ of the truncated EM solutions to the true solution. However, the convergence was in the asymptotic form without the convergence rate. In this section, we will discuss the rate. To avoid the notation becoming too complicated, we will only discuss the convergence rate in $L^{2}$ but the technique developed here can certainly be applied to study the rate in $L^{q}$. Recall that we use two functions $\mu(\cdot)$ and $h(\cdot)$ to define the truncated EM method. The choices of these functions are independent as long as they satisfy (2.5) and (2.6), respectively. It is interesting to see that they will satisfy a related condition in order for us to obtain the convergence rate.

We need an additional condition. To state it, we need a new notation. Let $\mathcal{U}$ denote the family of continuous functions $U: \mathbb{R}^{n} \times \mathbb{R}^{n} \rightarrow \mathbb{R}_{+}$such that for each $b>0$, there is a positive constant $\kappa_{b}$ for which

$$
U(x, \bar{x}) \leq \kappa_{b}|x-\bar{x}|^{2}, \quad \forall x, \bar{x} \in \mathbb{R}^{n} \text { with }|x| \vee|\bar{x}| \leq b .
$$

Assumption 5.1 Assume that there is a positive constant $H_{1}$ and a function $U \in \mathcal{U}$ such that

$$
\begin{aligned}
& (x-\bar{x})^{T}(f(x, y)-f(\bar{x}, \bar{y}))+\frac{1}{2}|g(x, y)-g(\bar{x}, \bar{y})|^{2} \\
\leq & H_{1}\left(|x-\bar{x}|^{2}+|y-\bar{y}|^{2}\right)-U(x, \bar{x})+U(y, \bar{y})
\end{aligned}
$$

for all $x, y, \bar{x}, \bar{y} \in \mathbb{R}^{n}$.

Let us first present a key lemma.

Lemma 5.2 Let Assumptions 2.1, 3.4 and 5.1 hold. Let $R>\|\xi\|$ be a real number and let $\Delta \in\left(0, \Delta^{*}\right)$ be sufficiently small such that $\mu^{-1}(h(\Delta)) \geq R$. Let $\theta_{\Delta, R}$ and $e_{\Delta}(t)$ be the same as defined in Section 3. Then,

$$
\mathbb{E}\left|e_{\Delta}\left(T \wedge \theta_{\Delta, R}\right)\right|^{2} \leq C\left(\Delta^{2 \gamma} \vee\left[\Delta^{1 / 2}(h(\Delta))^{2}\right]\right),
$$

where, as before, $C$ is the generic constant independent of $R$ and $\Delta$.

Proof We write $\theta_{\Delta, R}=\theta$ for simplicity. The Itô formula shows that

$$
\begin{aligned}
\mathbb{E}\left|e_{\Delta}(t \wedge \theta)\right|^{2}=\mathbb{E} \int_{0}^{t \wedge \theta}( & 2 e_{\Delta}^{T}(s)\left[f(x(s), x(s-\tau))-f_{\Delta}\left(\bar{x}_{\Delta}(s), \bar{x}_{\Delta}(s-\tau)\right)\right] \\
+ & \left.+g(x(s), x(s-\tau))-\left.g_{\Delta}\left(\bar{x}_{\Delta}(s), \bar{x}_{\Delta}(s-\tau)\right)\right|^{2}\right) d s
\end{aligned}
$$

for $0 \leq t \leq T$. We observe that for $0 \leq s \leq t \wedge \theta$,

$$
\left|\bar{x}_{\Delta}(s)\right| \vee\left|\bar{x}_{\Delta}(s-\tau)\right| \vee|x(s)| \vee|x(s-\tau)| \leq R .
$$

But we have the condition that $\mu^{-1}(h(\Delta)) \geq R$, so

$$
\left|\bar{x}_{\Delta}(s)\right| \vee\left|\bar{x}_{\Delta}(s-\tau)\right| \vee|x(s)| \vee|x(s-\tau)| \leq \mu^{-1}(h(\Delta)) .
$$


Recalling the definition of the truncated functions $f_{\Delta}$ and $g_{\Delta}$ as well as (2.5), we hence have that

$$
\begin{aligned}
f_{\Delta}\left(\bar{x}_{\Delta}(s), \bar{x}_{\Delta}(s-\tau)\right) & =f\left(\bar{x}_{\Delta}(s), \bar{x}_{\Delta}(s-\tau)\right), g_{\Delta}\left(\bar{x}_{\Delta}(s), \bar{x}_{\Delta}(s-\tau)\right) \\
& =g\left(\bar{x}_{\Delta}(s), \bar{x}_{\Delta}(s-\tau)\right)
\end{aligned}
$$

and

$$
|f(x(s), x(s-\tau))| \vee\left|f\left(\bar{x}_{\Delta}(s), \bar{x}_{\Delta}(s-\tau)\right)\right| \leq h(\Delta)
$$

for $0 \leq s \leq t \wedge \theta$. It therefore follows from (5.3) that

$$
\begin{aligned}
& \mathbb{E}\left|e_{\Delta}(t \wedge \theta)\right|^{2} \\
= & \mathbb{E} \int_{0}^{t \wedge \theta}\left(2 e_{\Delta}^{T}(s)\left[f(x(s), x(s-\tau))-f\left(\bar{x}_{\Delta}(s), \bar{x}_{\Delta}(s-\tau)\right)\right]\right. \\
& \left.\quad+\left|g(x(s), x(s-\tau))-g\left(\bar{x}_{\Delta}(s), \bar{x}_{\Delta}(s-\tau)\right)\right|^{2}\right) d s \\
= & \mathbb{E} \int_{0}^{t \wedge \theta}\left(2\left(x(s)-\bar{x}_{\Delta}(s)\right)^{T}\left[f(x(s), x(s-\tau))-f\left(\bar{x}_{\Delta}(s), \bar{x}_{\Delta}(s-\tau)\right)\right]\right. \\
& \left.\quad+\left|g(x(s), x(s-\tau))-g\left(\bar{x}_{\Delta}(s), \bar{x}_{\Delta}(s-\tau)\right)\right|^{2}\right) d s \\
& +\mathbb{E} \int_{0}^{t \wedge \theta} 2\left(\bar{x}_{\Delta}(s)-x_{\Delta}(s)\right)^{T}\left[f(x(s), x(s-\tau))-f\left(\bar{x}_{\Delta}(s), \bar{x}_{\Delta}(s-\tau)\right)\right] d s .
\end{aligned}
$$

By Assumption 5.1 and (5.4), we then derive that

$$
\begin{aligned}
\mathbb{E}\left|e_{\Delta}(t \wedge \theta)\right|^{2} \leq & 2 H_{1} \mathbb{E} \int_{0}^{t \wedge \theta}\left(\left|x(s)-\bar{x}_{\Delta}(s)\right|^{2}+\left|x(s-\tau)-\bar{x}_{\Delta}(s-\tau)\right|^{2}\right) d s \\
& +\mathbb{E} \int_{0}^{t \wedge \theta}\left(-U\left(x(s), \bar{x}_{\Delta}(s)\right)+U\left(x(s-\tau), \bar{x}_{\Delta}(s-\tau)\right)\right) d s \\
& +4 h(\Delta) \mathbb{E} \int_{0}^{t \wedge \theta}\left|\bar{x}_{\Delta}(s)-x_{\Delta}(s)\right| d s
\end{aligned}
$$

But, by Assumption 3.4 and Lemma 2.5, we derive that

$$
\begin{aligned}
& \quad \mathbb{E} \int_{0}^{t \wedge \theta}\left(\left|x(s)-\bar{x}_{\Delta}(s)\right|^{2}+\left|x(s-\tau)-\bar{x}_{\Delta}(s-\tau)\right|^{2}\right) d s \\
& \leq 2 \mathbb{E} \int_{0}^{t \wedge \theta}\left(\left|e_{\Delta}(s)\right|^{2}+\left|e_{\Delta}(s-\tau)\right|^{2}+\left|x_{\Delta}(s)-\bar{x}_{\Delta}(s)\right|^{2}\right. \\
& \left.\quad+\left|x_{\Delta}(s-\tau)-\bar{x}_{\Delta}(s-\tau)\right|^{2}\right) d s \\
& \leq 4 \mathbb{E} \int_{0}^{t}\left|e_{\Delta}(s \wedge \theta)\right|^{2} d s+4 \int_{0}^{T} \mathbb{E}\left|x_{\Delta}(s)-\bar{x}_{\Delta}(s)\right|^{2} d s \\
& \quad+\int_{-\tau}^{0}|\xi(s)-\xi(\lfloor s / \Delta\rfloor \Delta)|^{2} d s \\
& \leq 4 \int_{0}^{t} \mathbb{E}\left|e_{\Delta}(s \wedge \theta)\right|^{2} d s+C \Delta(h(\Delta))^{2}+\tau K_{3}^{2} \Delta^{2 \gamma} .
\end{aligned}
$$


Moreover, by the property of the $\mathcal{U}$-class function $U$ and Assumption 3.4, we have

$$
\begin{aligned}
& \mathbb{E} \int_{0}^{t \wedge \theta}\left(-U\left(x(s), \bar{x}_{\Delta}(s)\right)+U\left(x(s-\tau), \bar{x}_{\Delta}(s-\tau)\right)\right) d s \\
\leq & \int_{-\tau}^{0} U(\xi(s), \xi(\lfloor s / \Delta\rfloor \Delta)) d s \leq \int_{-\tau}^{0} \kappa_{b}|\xi(s)-\xi(\lfloor s / \Delta\rfloor \Delta)|^{2} d s \\
\leq & \tau \kappa_{b} K_{3}^{2} \Delta^{2 \gamma},
\end{aligned}
$$

where $b=\|\xi\|$. Furthermore, by Lemma 2.5,

$$
\mathbb{E} \int_{0}^{t \wedge \theta}\left|\bar{x}_{\Delta}(s)-x_{\Delta}(s)\right| d s \leq \int_{0}^{T} \mathbb{E}\left|\bar{x}_{\Delta}(s)-x_{\Delta}(s)\right| d s \leq C \Delta^{1 / 2} h(\Delta) .
$$

Substituting (5.7)-(5.9) into (5.6), we get

$$
\mathbb{E}\left|e_{\Delta}(t \wedge \theta)\right|^{2} \leq 8 H_{1} \int_{0}^{t} \mathbb{E}\left|e_{\Delta}(s \wedge \theta)\right|^{2} d s+C\left(\Delta^{2 \gamma} \vee\left[\Delta^{1 / 2}(h(\Delta))^{2}\right]\right)
$$

By the Gronwall inequality, we obtain the required assertion (5.2).

Let us now state our first result on the convergence rate, where we reveal a strong relation between functions $\mu(\cdot)$ and $h(\cdot)$, which are used to define the truncated EM method.

Theorem 5.3 Let Assumptions 2.1, 5.1, 4.1 and 3.4 hold. Assume that

$$
h(\Delta) \geq \mu\left(\left(\Delta^{2 \gamma} \vee\left[\Delta^{1 / 2}(h(\Delta))^{2}\right]\right)^{-1 /(\bar{p}-2)}\right)
$$

for all sufficiently small $\Delta \in\left(0, \Delta^{*}\right)$. Then, for every such small $\Delta$,

$$
\mathbb{E}\left|x(T)-x_{\Delta}(T)\right|^{2} \leq C\left(\Delta^{2 \gamma} \vee\left[\Delta^{1 / 2}(h(\Delta))^{2}\right]\right)
$$

and

$$
\mathbb{E}\left|x(T)-\bar{x}_{\Delta}(T)\right|^{2} \leq C\left(\Delta^{2 \gamma} \vee\left[\Delta^{1 / 2}(h(\Delta))^{2}\right]\right) .
$$

Proof We use the same notation as in the proof of Theorem 4.7. It follows from (4.9)-(4.11) with $q=2$ that the inequality

$$
\mathbb{E}\left|e_{\Delta}(T)\right|^{2} \leq \mathbb{E}\left(\left|e_{\Delta}\left(T \wedge \theta_{\Delta, R}\right)\right|^{2}\right)+\frac{2 C \delta}{\bar{p}}+\frac{C(\bar{p}-2)}{\bar{p} R^{\bar{p}} \delta^{2 /(\bar{p}-2)}}
$$

holds for any $\Delta \in\left(0, \Delta^{*}\right), R>\|\xi\|$ and $\delta>0$. In particular, choosing

$$
\delta=\Delta^{2 \gamma} \vee\left[\Delta^{1 / 2}(h(\Delta))^{2}\right] \quad \text { and } R=\left(\Delta^{2 \gamma} \vee\left[\Delta^{1 / 2}(h(\Delta))^{2}\right]\right)^{-1 /(\bar{p}-2)},
$$

we get

$$
\mathbb{E}\left|e_{\Delta}(T)\right|^{2} \leq \mathbb{E}\left|e_{\Delta}\left(T \wedge \theta_{\Delta, R}\right)\right|^{2}+C\left(\Delta^{2 \gamma} \vee\left[\Delta^{1 / 2}(h(\Delta))^{2}\right]\right)
$$

But, by condition (5.10), we have

$$
\mu^{-1}(h(\Delta)) \geq\left(\Delta^{2 \gamma} \vee\left[\Delta^{1 / 2}(h(\Delta))^{2}\right]\right)^{-1 /(\bar{p}-2)}=R
$$

We can hence apply Lemma 5.2 to obtain

$$
\mathbb{E}\left|e_{\Delta}\left(T \wedge \theta_{\Delta, R}\right)\right|^{2} \leq C\left(\Delta^{2 \gamma} \vee\left[\Delta^{1 / 2}(h(\Delta))^{2}\right]\right)
$$


Substituting this into (5.14) yields the first assertion (5.11). The second assertion (5.12) follows from (5.11) and Lemma 2.5.

Let us discuss an example to illustrate Theorem 5.3 and to motivate our further results on the convergence rates.

Example 5.4 Consider the same SDE in Example 4.8. We need to verify Assumption 5.1. For $x, y, \bar{x}, \bar{y} \in \mathbb{R}$, it is easy to show that

$$
(x-\bar{x})(f(x, y)-f(\bar{x}, \bar{y})) \leq a_{2}^{2}|x-\bar{x}|^{2}+\left(|y|^{4 / 3}-|\bar{y}|^{4 / 3}\right)^{2}-0.5 a_{3}|x-\bar{x}|^{2}\left(x^{2}+\bar{x}^{2}\right) \text {. }
$$

But, by the mean value theorem,

$$
\left(|y|^{4 / 3}-|\bar{y}|^{4 / 3}\right)^{2} \leq \frac{16}{9}|y-\bar{y}|^{2}\left(|y|^{1 / 3}+|\bar{y}|^{1 / 3}\right)^{2} \leq 4|y-\bar{y}|^{2}\left(|y|^{2 / 3}+|\bar{y}|^{2 / 3}\right) .
$$

Let $a_{6}:=\sup _{u \geq 0}\left(8 u^{2 / 3}-0.5 a_{3} u^{2}\right)$. Then $0 \leq a_{6}<\infty$ and

$$
\left(|y|^{4 / 3}-|\bar{y}|^{4 / 3}\right)^{2} \leq a_{6}|y-\bar{y}|^{2}+0.25 a_{3}|y-\bar{y}|^{2}\left(y^{2}+\bar{y}^{2}\right) .
$$

Substituting this into (5.16) yields

$$
\begin{aligned}
& (x-\bar{x})(f(x, y)-f(\bar{x}, \bar{y})) \\
\leq & \left(a_{6} \vee a_{2}^{2}\right)\left(|x-\bar{x}|^{2}+|y-\bar{y}|^{2}\right) \\
& -0.5 a_{3}|x-\bar{x}|^{2}\left(x^{2}+\bar{x}^{2}\right)+0.25 a_{3}|y-\bar{y}|^{2}\left(y^{2}+\bar{y}^{2}\right) .
\end{aligned}
$$

Similarly, we can show that

$0.5|g(x, y)-g(\bar{x}, \bar{y})|^{2} \leq\left(a_{7} \vee a_{5}^{2}\right)\left(|x-\bar{x}|^{2}+|y-\bar{y}|^{2}\right)+0.25 a_{3}|x-\bar{x}|^{2}\left(x^{2}+\bar{x}^{2}\right)$,

where $a_{7}:=\sup _{u \geq 0}\left(9 a_{4}^{2} u-0.5 a_{3} u^{2}\right) \in(0, \infty)$. It then follows from (5.17) and (5.18) that

$$
\begin{aligned}
& (x-\bar{x})(f(x, y)-f(\bar{x}, \bar{y}))+0.5|g(x, y)-g(\bar{x}, \bar{y})|^{2} \\
\leq & H_{1}\left(|x-\bar{x}|^{2}+|y-\bar{y}|^{2}\right)-U(x, \bar{x})+U(y, \bar{y}),
\end{aligned}
$$

where $H_{1}=\left(a_{6} \vee a_{2}^{2}\right)+\left(a_{7} \vee a_{5}^{2}\right)$ and $U(x, \bar{x})=0.25 a_{3}|x-\bar{x}|^{2}\left(x^{2}+\bar{x}^{2}\right)$. It is obvious that $U \in \mathcal{U}$. In other words, we have shown that Assumption 5.1 is satisfied too. To apply Theorem 5.3, we use the same functions $\mu(\cdot)$ and $h(\cdot)$ as defined in Example 4.8. We observe that inequality (5.10) becomes

$$
\Delta^{-\varepsilon} \geq \hat{a} \Delta^{-3[(2 \gamma) \wedge(1 / 2-2 \varepsilon)] /(\bar{p}-2)} .
$$

But, for any $\varepsilon \in(0,1 / 4]$, we can choose $\bar{p}$ sufficiently large such that $\varepsilon>3[(2 \gamma) \wedge$ $(1 / 2-2 \varepsilon)] /(\bar{p}-2)$ and hence $(5.20)$ holds for all sufficiently small $\Delta$. We can therefore conclude by Theorem 5.3 that the truncated EM solutions of the SDE (4.14) satisfy

$\mathbb{E}\left|x(T)-x_{\Delta}(T)\right|^{2}=O\left(\Delta^{(2 \gamma) \wedge(1 / 2-2 \varepsilon)}\right)$ and $\mathbb{E}\left|x(T)-\bar{x}_{\Delta}(T)\right|^{2}=O\left(\Delta^{(2 \gamma) \wedge(1 / 2-2 \varepsilon)}\right)$.

It is known that for every $\alpha \in(0,0.5)$, the Brownian motion is $\alpha$-Hölder continuous (see, e.g., [10]). If we regard the initial data $\xi(u), u \in[-\tau, 0]$ as an observation of 
the state during the time interval $[-\tau, 0]$, it is reasonable to assume that $\gamma \in(0,0.5)$. If $\gamma$ is close to 0.5 , then (5.21) shows the order of convergence is close to 0.25 . Can we improve the order? The answer is yes though we need stronger conditions.

Assumption 5.5 Assume that there are positive constants $\alpha$ and $\mathrm{H}_{2}$ and a function $U \in \mathcal{U}$ such that

$$
\begin{aligned}
& (x-\bar{x})^{T}(f(x, y)-f(\bar{x}, \bar{y}))+\frac{1+\alpha}{2}|g(x, y)-g(\bar{x}, \bar{y})|^{2} \\
\leq & H_{2}\left(|x-\bar{x}|^{2}+|y-\bar{y}|^{2}\right)-U(x, \bar{x})+U(y, \bar{y})
\end{aligned}
$$

for all $x, y, \bar{x}, \bar{y} \in \mathbb{R}^{n}$.

Assumption 5.6 Assume that there is a pair of positive constants $r$ and $\mathrm{H}_{3}$ such that

$$
\begin{aligned}
& |f(x, y)-f(\bar{x}, \bar{y})|^{2} \vee|g(x, y)-g(\bar{x}, \bar{y})|^{2} \\
\leq & H_{3}\left(|x-\bar{x}|^{2}+|y-\bar{y}|^{2}\right)\left(1+|x|^{r}+|\bar{x}|^{r}+|y|^{r}+|\bar{y}|^{r}\right)
\end{aligned}
$$

for all $x, y, \bar{x}, \bar{y} \in \mathbb{R}^{n}$.

Lemma 5.7 Let Assumptions 2.1, 3.4, 4.1, 4.1 and 3.4 hold and $\bar{p}>$ r. Let $R>\|\xi\|$ be a real number and let $\Delta \in\left(0, \Delta^{*}\right)$ be sufficiently small such that $\mu^{-1}(h(\Delta)) \geq R$. Let $\theta_{\Delta, R}$ and $e_{\Delta}(t)$ be the same as defined in Section 3. Then

$$
\mathbb{E}\left|e_{\Delta}\left(T \wedge \theta_{\Delta, R}\right)\right|^{2} \leq C\left(\Delta^{2 \gamma} \vee\left[\Delta(h(\Delta))^{2}\right]\right)
$$

Proof We use the same notation as in the proof of Lemma 5.2. It follows from (5.5) that

$$
\begin{aligned}
\mathbb{E}\left|e_{\Delta}(t \wedge \theta)\right|^{2} \leq & \mathbb{E} \int_{0}^{t \wedge \theta}\left(2 e_{\Delta}^{T}(s)\left[f(x(s), x(s-\tau))-f\left(x_{\Delta}(s), x_{\Delta}(s-\tau)\right)\right]\right. \\
& +(1+\alpha)\left|g(x(s), x(s-\tau))-g\left(x_{\Delta}(s), x_{\Delta}(s-\tau)\right)\right|^{2} \\
& +2 e_{\Delta}^{T}(s)\left[f\left(x_{\Delta}(s), x_{\Delta}(s-\tau)\right)-f\left(\bar{x}_{\Delta}(s), \bar{x}_{\Delta}(s-\tau)\right)\right] \\
& \left.+\left(1+\alpha^{-1}\right)\left|g\left(x_{\Delta}(s), x_{\Delta}(s-\tau)\right)-g\left(\bar{x}_{\Delta}(s), \bar{x}_{\Delta}(s-\tau)\right)\right|^{2}\right) d s .
\end{aligned}
$$

By Assumptions 3.4, 4.1 and 3.4, we can then show

$$
\mathbb{E}\left|e_{\Delta}(t \wedge \theta)\right|^{2} \leq\left(4 H_{2}+1\right) \int_{0}^{t} \mathbb{E}\left|e_{\Delta}(s \wedge \theta)\right|^{2} d s+2 \tau \kappa_{b} K_{3}^{2} \Delta^{2 \gamma}+J,
$$

where (5.8) has been used and

$$
\begin{aligned}
J:= & \mathbb{E} \int_{0}^{t \wedge \theta} H_{3}\left(2+\alpha^{-1}\right)\left(\left|x_{\Delta}(s)-\bar{x}_{\Delta}(s)\right|^{2}+\left|x_{\Delta}(s-\tau)-\bar{x}_{\Delta}(s-\tau)\right|^{2}\right) \\
& \times\left(1+\left|x_{\Delta}(s)\right|^{r}+\left|\bar{x}_{\Delta}(s)\right|^{r}+\left|x_{\Delta}(s-\tau)\right|^{r}+\left|\bar{x}_{\Delta}(s-\tau)\right|^{r}\right) d s .
\end{aligned}
$$


But, by the Hölder inequality, Lemmas 2.5 and 4.3 and Assumption 3.4, we can derive that

$$
\begin{aligned}
J \leq & C \int_{0}^{T}\left(\mathbb{E}\left|x_{\Delta}(s)-\bar{x}_{\Delta}(s)\right|^{2 \bar{p} /(\bar{p}-r)}+\mathbb{E}\left|x_{\Delta}(s-\tau)-\bar{x}_{\Delta}(s-\tau)\right|^{2 \bar{p} /(\bar{p}-r)}\right)^{(\bar{p}-r) / \bar{p}} \\
& \times\left(1+\mathbb{E}\left|x_{\Delta}(s)\right|^{\bar{p}}+\mathbb{E}\left|\bar{x}_{\Delta}(s)\right|^{\bar{p}}+\mathbb{E}\left|x_{\Delta}(s-\tau)\right|^{\bar{p}}+\mathbb{E}\left|\bar{x}_{\Delta}(s-\tau)\right|^{\bar{p}}\right)^{r / \bar{p}} d s \\
\leq & C\left(\Delta^{2 \gamma} \vee\left[\Delta(h(\Delta))^{2}\right]\right) .
\end{aligned}
$$

Substituting this into (5.26) gives

$$
\mathbb{E}\left|e_{\Delta}(t \wedge \theta)\right|^{2} \leq\left(4 H_{2}+1\right) \int_{0}^{t} \mathbb{E}\left|e_{\Delta}(s \wedge \theta)\right|^{2} d s+C\left(\Delta^{2 \gamma} \vee\left[\Delta(h(\Delta))^{2}\right]\right),
$$

which implies the required assertion (5.24).

The following theorem gives a better convergence rate than Theorem 5.3.

Theorem 5.8 Let Assumptions 2.1, 3.4, 4.1, 4.1 and 3.4 hold and $\bar{p}>r$. Assume that

$$
h(\Delta) \geq \mu\left(\left(\Delta^{2 \gamma} \vee\left[\Delta(h(\Delta))^{2}\right]\right)^{-1 /(\bar{p}-2)}\right)
$$

for all sufficiently small $\Delta \in\left(0, \Delta^{*}\right)$. Then, for every such small $\Delta$,

$$
\mathbb{E}\left|x(T)-x_{\Delta}(T)\right|^{2} \leq C\left(\Delta^{2 \gamma} \vee\left[\Delta(h(\Delta))^{2}\right]\right)
$$

and

$$
\mathbb{E}\left|x(T)-\bar{x}_{\Delta}(T)\right|^{2} \leq C\left(\Delta^{2 \gamma} \vee\left[\Delta(h(\Delta))^{2}\right]\right) .
$$

Proof We use the same notation as in the proof of Theorem 5.3. Choosing

$$
\delta=\Delta^{2 \gamma} \vee\left[\Delta(h(\Delta))^{2}\right] \quad \text { and } \quad R=\left(\Delta^{2 \gamma} \vee\left[\Delta(h(\Delta))^{2}\right]\right)^{-1 /(\bar{p}-2)},
$$

we get from (5.13) that

$$
\mathbb{E}\left|e_{\Delta}(T)\right|^{2} \leq \mathbb{E}\left|e_{\Delta}\left(T \wedge \theta_{\Delta, R}\right)\right|^{2}+C\left(\Delta^{2 \gamma} \vee\left[\Delta(h(\Delta))^{2}\right]\right) .
$$

But, by condition (5.27), we have

$$
\mu^{-1}(h(\Delta)) \geq\left(\Delta^{2 \gamma} \vee\left[\Delta(h(\Delta))^{2}\right]\right)^{-1 /(\bar{p}-2)}=R .
$$

We can hence apply Lemma 5.2 to obtain

$$
\mathbb{E}\left|e_{\Delta}\left(T \wedge \theta_{\Delta, R}\right)\right|^{2} \leq C\left(\Delta^{2 \gamma} \vee\left[\Delta(h(\Delta))^{2}\right]\right) .
$$

Substituting this into (5.30) yields the first assertion (5.28). The second assertion (5.29) follows from (5.28) and Lemma 2.5.

Example 5.9 Let us return to Example 4.8 once again. Instead of (5.18), we can have the following alternative estimate $|g(x, y)-g(\bar{x}, \bar{y})|^{2} \leq 2\left(a_{8} \vee a_{5}^{2}\right)\left(|x-\bar{x}|^{2}+|y-\bar{y}|^{2}\right)+0.25 a_{3}|x-\bar{x}|^{2}\left(x^{2}+\bar{x}^{2}\right)$, 
where $a_{8}:=\sup _{u \geq 0}\left(9 a_{4}^{2} u-0.25 a_{3} u^{2}\right) \in(0, \infty)$. It then follows from (5.17) and (5.32) that

$$
\begin{aligned}
& (x-\bar{x})(f(x, y)-f(\bar{x}, \bar{y}))+|g(x, y)-g(\bar{x}, \bar{y})|^{2} \\
\leq & H_{2}\left(|x-\bar{x}|^{2}+|y-\bar{y}|^{2}\right)-U(x, \bar{x})+U(y, \bar{y}),
\end{aligned}
$$

where $H_{2}=\left(a_{6} \vee a_{2}^{2}\right)+2\left(a_{8} \vee a_{5}^{2}\right)$ and $U(x, \bar{x})=0.25 a_{3}|x-\bar{x}|^{2}\left(x^{2}+\bar{x}^{2}\right)$. In other words, we have shown that Assumption 4.1 is satisfied with $\alpha=1$. It is also straightforward to show that

$$
|f(x, y)-f(\bar{x}, \bar{y})|^{2} \leq 8 a_{2}^{2}|y-\bar{y}|^{2}\left(1+|y|^{4}+|\bar{y}|^{4}\right)+16 a_{3}^{2}|x-\bar{x}|^{2}\left(|x|^{4}+|\bar{x}|^{4}\right) .
$$

We hence see from (5.32) and (5.34) that Assumption 3.4 is also satisfied with $r=4$. In other words, we have shown that Assumptions 2.1, 4.1, 3.4, 4.1, and 3.4 hold for every $\bar{p}>r=4$. Let $\mu(\cdot)$ and $h(\cdot)$ be the same as before. We can then conclude by Theorem 5.8 that the truncated EM solutions of the SDE (4.14) satisfy

$\mathbb{E}\left|x(T)-x_{\Delta}(T)\right|^{2}=O\left(\Delta^{(2 \gamma) \wedge(1-2 \varepsilon)}\right)$ and $\mathbb{E}\left|x(T)-\bar{x}_{\Delta}(T)\right|^{2}=O\left(\Delta^{(2 \gamma) \wedge(1-2 \varepsilon)}\right)$.

In particular, if $\gamma$ is close to 0.5 (or bigger than half), this shows that the order of convergence is close to 0.5 .

\section{Conclusion}

In this paper, we have used the new explicit method, called the truncated EM method, to study the strong convergence of the numerical solutions for nonlinear SDDEs. For a given stepsize $\Delta$, we define the discrete-time truncated EM numerical solution and then form two versions of the continuous-time truncated EM solutions, namely the continuous-time step-process truncated EM solution $\bar{x}_{\Delta}(t)$ and the continuous-time continuous-process truncated EM solution $x_{\Delta}(t)$. Under the local Lipschitz condition plus the generalized Khasminskii-type condition, we have successfully shown the strong convergence of both continuous-time truncated EM solutions to the true solution in the sense that

$$
\lim _{\Delta \rightarrow 0} \mathbb{E}\left|x_{\Delta}(T)-x(T)\right|^{q}=0 \quad \text { and } \quad \lim _{\Delta \rightarrow 0} \mathbb{E}\left|\bar{x}_{\Delta}(T)-x(T)\right|^{q}=0
$$

for any $T>0$ and $q \in[1,2)$. Under a slightly stronger Khasminskii-type condition, we have showed the above convergence for some $q \geq 2$. We have also discussed the convergence rates in $L^{2}$ under some additional conditions. We have used several examples to illustrate our theory throughout the paper.

Acknowledgements The authors would like to thank the associate editor and referees for their very helpful comments and suggestions. The authors would also like to thank the Leverhulme Trust (RF-2015-385), the Royal Society (WM160014, Royal Society Wolfson Research Merit Award), the Royal Society and the Newton Fund (NA160317, Royal Society-Newton Advanced Fellowship), the Natural Science Foundation of China (11471216), the Natural Science Foundation of Shanghai (14ZR1431300), the Ministry of Education (MOE) of China (MS2014DHDX020) for their financial support. 
Open Access This article is distributed under the terms of the Creative Commons Attribution 4.0 International License (http://creativecommons.org/licenses/by/4.0/), which permits unrestricted use, distribution, and reproduction in any medium, provided you give appropriate credit to the original author(s) and the source, provide a link to the Creative Commons license, and indicate if changes were made.

\section{References}

1. Arnold, L.: Stochastic differential equations, theory and applications. Wiley (1975)

2. Bahar, A., Mao, X.: Persistence of stochastic power law logistic model. J. Appl. Probab. Stat. 3(1), 37-43 (2008)

3. Baker, C.T.H., Buckwar, E.: Numerical analysis of explicit one-step methods for stochastic delay differential equations. LMS J. Comput. Math. 3, 315-335 (2000)

4. Baker, C.T.H., Buckwar, E.: Exponential stability in p-th mean of solutions, and of convergent Eulertype solutions, of stochastic delay differential equations. J. Comput. Appl. Math. 184, 404-427 (2005)

5. Caraballo, T., Kloeden, P.E., Real, J.: Discretization of asymptotically stable stationary solutions of delay differential equations with a random stationary delay. J. Dyn. Diff. Eqns. 18, 863-880 (2006)

6. Da Prato, G., Zabczyk, J.: Stochastic equations in infinite dimensions. Cambridge Univeristy Press (1992)

7. Khasminskii, R.Z.: Stochastic stability of differential equations. Sijtjoff and Noordhoff, Alphen (1980). (Translation of the Russian edition, Moscow, Nauka 1969)

8. Kloeden, P.E., Platen, E.: Numerical solution of stochastic differential equations. Springer-Verlag, New York (1992)

9. Kolmanovskii, V., Myshkis, A.: Applied theory of functional differential equations. Kluwer Academic Publishers (1992)

10. Karatzas, I., Shreve, S.E.: Brownian motion and stochastic calculus. Springer (1988)

11. Küchler, U., Platen, E.: Strong discrete time approximation of stochastic differential equations with time delay. Math. Comput. Simul. 54, 189-205 (2000)

12. Ladde, G.S., Lakshmikantham, V.: Ramdom differential inequalities. Academic Press (1980)

13. Mao, X. Stochastic differential equations and applications, 2nd edn. Horwood Publishing, Chichester (2007)

14. Mao, X.: A note on the LaSalle-type theorems for stochastic differential delay equations. J. Math. Anal. Appl. 268, 125-142 (2002)

15. Mao, X.: Numerical solutions of stochastic differential delay equations under the generalized Khasminskii-type conditions. Appl. Math. Comput. 217, 5512-5524 (2011)

16. Mao, X.: The truncated Euler-Maruyama method for stochastic differential equations. J. Comput. Appl. Math. 290, 370-384 (2015)

17. Mao, X., Rassias, M.J.: Khasminskii-type theorems for stochastic differential delay equations. J. Sto. Anal. Appl. 23, 1045-1069 (2005)

18. Mao, X., Sabanis, S.: Numerical solutions of stochastic differential delay equations under local Lipschitz condition. J. Comput. Appl Math. 151, 215-227 (2003)

19. Milstein, G.N.: Numerical integration of stochastic differential equations. Kluwer Academic Publishers, Dodrecht (1995)

20. Mohammed, S.-E.A.: Stochastic functional differential equations. Longman Scientific and Technical (1985)

21. Schurz, H.: Applications of numerical methods and its analysis for systems of stochastic differential equations. Bull. Kerala Math. Assoc. 4, 1-85 (2007)

22. Wu, F., Mao, X.: Numerical solutions of neutral stochastic functional differential equations. SIAM J. Numer. Anal. 46(4), 1821-1841 (2008) 